OPEN ACCESS

Edited by:

Fengguo $\mathrm{Xu}$,

China Pharmaceutical University,

China

Reviewed by:

Chu Chu,

Zhejiang University of Technology,

China

Ruili Yang,

South China Agricultural University,

China

Wei Zhang,

Macau University of Science and

Technology, Macau

${ }^{*}$ Correspondence:

Yan-xu Chang

Tcmcyx@tjutcm.edu.cn

Specialty section:

This article was submitted to

Ethnopharmacology,

a section of the journal

Frontiers in Pharmacology

Received: 24 September 2019

Accepted: 21 May 2020

Published: 17 June 2020

Citation:

Guo J, Li J, Yang X, Wang H, He J,

Liu E, Gao X and Chang Y-x (2020) A

Metabolomics Coupled With

Chemometrics Strategy to Filter

Combinatorial Discriminatory Quality

Markers of Crude and Salt-Fired

Eucommiae Cortex.

Front. Pharmacol. 11:838.

doi: 10.3389/fphar.2020.00838

\section{A Metabolomics Coupled With Chemometrics Strategy to Filter Combinatorial Discriminatory Quality Markers of Crude and Salt-Fired Eucommiae Cortex}

\author{
Jiading Guo ${ }^{1,2}$, Jin $\mathrm{Li}^{1}$, Xuejing Yang ${ }^{1,3}$, Hui Wang ${ }^{1,2}$, Jun He ${ }^{1,2}$, Erwei Liu ${ }^{1,2}$, \\ Xiumei Gao ${ }^{1,2}$ and Yan-xu Chang ${ }^{1,2^{*}}$ \\ 1 Tianjin State Key Laboratory of Modern Chinese Medicine, Tianjin University of Traditional Chinese Medicine, Tianjin, China, \\ 2 Tianjin Key Laboratory of Phytochemistry and Pharmaceutical Analysis, Tianjin University of Traditional Chinese Medicine, \\ Tianjin, China, ${ }^{3}$ School of Pharmacy, Harbin University of Commerce, Harbin, China
}

Eucommiae Cortex is commonly used for treating various diseases in a form of the crude and salt-fired products. Generally, it is empirical to distinguish the difference between two types of Eucommiae Cortex. The metabolomics coupled with chemometrics strategy was proposed to filter the combinatorial discriminatory quality markers for precise distinction and further quality control of the crude and salt-fired Eucommiae Cortex. The metabolomics data of multiple batches of Eucommiae Cortex samples was obtained by ultra-high performance liquid chromatography coupled with mass spectrometry (UHPLCMS). Orthogonal partial least-squares discriminant analysis was utilized to filter candidate markers for characterizing the obvious difference of the crude and salt-fired Eucommiae Cortex. The accuracy of combinatorial markers was validated by random forest and partial least squares regression. Finally, eleven combinatorial discriminatory quality markers from 67 identified compounds were rapidly screened, identified, and determined for distinguishing the difference between crude and salt-fired Eucommiae Cortex. It was demonstrated that UHPLC-MS based metabolomics with chemometrics was a powerful strategy to screen the combinatorial discriminatory quality markers for distinguishing the crude and salt-fired Eucommiae Cortex and to provide the reference for precise quality control of Eucommiae Cortex.

Keywords: Eucommiae Cortex, ultra-high performance liquid chromatography coupled with mass spectrometry, metabolomics, chemometrics, combinatorial discriminatory quality markers

\section{INTRODUCTION}

Eucommiae Cortex, also named Duzhong in China, is the dry bark of Eucommia ulmoides Oliv. tree and one of the oldest traditional chinese herbal medicines (Zhao et al., 2015). It has been listed as one of the "Middle grade" medicines in Sheng Nong's herbal classic since two thousand years ago (Cronquist and Takhtadzhian, 1981). It is used clinically to treat a variety of diseases such as 
osteoporosis, rheumatoid arthritis, hypertension, and menopause syndrome (He et al., 2014). The active ingredients mainly included lignans, iridoids, phenolics, and so on. These ingredients have a wide range of pharmacological activities such as antihypertensive, anti-aging, antioxidant, antimutagenic, and anti-inflammatory activities (Li et al., 2014; Zhu and Sun, 2018).

Traditional Chinese Herbs (TCHs) have been widely used to treat various diseases over thousand years and its global demand is also increasing year after year. Generally, most of Chinese herbs should be prepared in several special processing ways such as stir-frying, steaming, boiling, stewing, and so on (Wang F. et al., 2017). This may directly change the content of some certain compounds, possibly affecting the pharmacological activities of TCHs (Wu et al., 2018). As officially recorded in Chinese pharmacopeia (2015 edition), both the crude and saltfired Eucommiae Cortex commonly used to treat the disease in clinic. Moreover, salt-fired herb medicines are more preferred to act on the "kidney channel" and further improve kidney and liver function according to the Chinese medicine traditional processing theory. Modern research showed that the content and absorption behavior of active compounds would be obviously changed when the Eucommiae Cortex was subject to the salt-fired processing (Lu et al., 2018). Importantly, it needs to be clearly stated whether the crude or the salt-fired Eucommiae Cortex is used for Chinese medicine prescriptions. For example, the salt-fired Eucommiae Cortex was explicitly prescribed to be used for Yougui Wan, Tianma Wan and Qing'e Wan. Compared with the chemical drugs, herbal medicines are the mixtures of multicompounds, which would bring a huge challenge for prescribing the appropriate compounds for quality evaluation. The chemical marker of quality control (QC) of TCHs is commonly one or a few compound (Li et al., 2019). In Chinese Pharmacopeia (2015 edition), the quality standard of Eucommiae Cortex is that the content of pinoresinol di-o-glucopyranoside is not less than $0.1 \%$. However, it may be not specific and practical due to a lack of definitive standard used for distinguishing two types of Eucommiae Cortex products. Therefore, it is necessary to discover the effective quality markers for distinguishing the crude and salt-fired Eucommiae Cortex.

Recently, metabolomic technology has become an important and valuable tool in the life sciences. It has been extended to a variety of research areas such as biomarker discovery, disease diagnosis, and quality evaluation of TCHs (Mao et al., 2017; Aszyk et al., 2018). Fortunately, metabolomic methods greatly contribute to discoveries of difference markers that represent the change in the biological environment caused by the special disturbances. Liquid chromatography-mass spectrometry (LCMS) method plays an important role in the acquisition of metabolomic dataset and identification of metabolites depending on its high separation capacity and sensitivity (Zhou et al., 2012). Chemometrics is quite versatile due to the perfect combination of mathematics, statistics, and computer science (Ziegel, 2004). It provides many good algorithms to mine and retrieve more valuable chemical information from natural products (Kumar et al., 2014). Among the algorithms, random forest $(\mathrm{RF})$ and partial least squares regression (PLSR) are commonly regarded as the effective tool in classification and accuracy prediction for multivariate data (Xia et al., 2017; Wang et al., 2019). Thus, the use of metabolomics in combination with chemometrics might exhibit the unique advantage for the analysis the discrimination between crude Eucommiae Cortex and its processed product.

In this work, an LC-MS based metabolomics coupled with chemometrics strategy was proposed to screen the combinatorial discriminatory quality markers (CdQMs) for distinction of crude and salt-fired Eucommiae Cortex. Firstly, a total of the 38 different batches of the crude and salt-fired Eucommiae Cortex were subjected to ultra-high performance liquid chromatography coupled with quadrupole time-of-flight mass spectrometry (UHPLC-Q-TOF/MS) analysis for acquisition of the whole chemical profile. Secondly, the CdQMs were stepwise filtered from massive metabolomics data by a series of approaches of chemometrics analysis. To be specific, the filtering process was followed by the several rules: 1) the markers could well distinguish the crude and salt-fired Eucommiae Cortex; 2) the markers had high accuracies; 3 ) the markers were easy to access commercially and quantify. At last, the content of the CdQMs in the crude and salt-fired Eucommiae Cortex were analyzed by UHPLC-PDA (photodiode array detector) method and the effectiveness of CdQMs were further validated by discriminant analysis. The LC-MS metabolomics coupled with chemometrics strategy was successfully used to screen the CdQMs for distinguishing the crude and salt-fired Eucommiae Cortex.

\section{MATERIALS AND METHODS}

\section{Plant Material}

A total of 54 batches of crude and salt-fired Eucommiae Cortex were used for this study. Among the, the 27 batches of crude samples (C1-C19 and VC1-VC8) and 19 batches of salt-fired samples (S1-S19) were purchased from different drugstores in Tianjin and Hebei province of China. Moreover, according to Chinese Pharmacopeia (2015 edition), we processed eight batches of salt-fired samples (VS1-VS8) using the crude samples (VC1-VC8). All samples were authenticated as Eucommia ulmoides Oliver. by Prof. Lin Ma (Tianjin University of Traditional Chinese Medicine). The voucher specimens of Eucommia ulmoides Oliv., such as QFNU QFNU0018228, QFNU QFNU0018229, SYS SYS00189991, WUK 0060451, etc., were deposited in Chinese Virtual Herbarium (http://www.cvh.ac.cn/), and corresponding herbarium codes, for example, QFNU, SYS, WUK, etc., were searchable in the NYBG Steere Herbarium (http://sweetgum. nybg.org/science/ih/?_ga=2.40299874.1384373005.15579301481052084957.1548409239).

\section{Chemicals and Reagents}

HPLC grade acetonitrile, methanol, and formic acid were obtained from Fisher Scientific (Pittsburg, PA, USA) and Anaqua $^{\mathrm{TM}}$ Chemicals Supply (Wilmington, DE, USA), respectively. Deionized water was purified by Milli-Q academic 
ultra-pure water system (Millipore, Milford, MA, USA). Standard substances such as geniposidic acid, neochlorogenic acid, chlorogenic acid, caffeic acid, geniposide, genipin, pinoresinol di-o-glucopyranoside, syringaresinol di-oglucopyranoside, isochlorogenic acid A, pinoresinol $o$ glucopyranoside, and isochlorogenic acid $\mathrm{C}$ were purchased from Chengdu Desite Bio-Technology Co., Ltd (Chengdu, China). The purity of all standard substances was more than $98 \%$.

\section{Preparation of Sample and Standard Substance Solution \\ Preparation of Sample Solution}

The samples were powered and passed through 80 mesh sieves. The powder $(0.400 \mathrm{~g})$ was accurately weighed and was then extracted by ultrasonic method $(40 \mathrm{kHz}, 1,200 \mathrm{~W})$ for $20 \mathrm{~min}$ at room temperature $\left(28^{\circ} \mathrm{C}\right)$ with $50 \%$ methanol-water $(10 \mathrm{ml})$. All the sample solution was passed through a $0.22-\mu \mathrm{m}$ filter membrane and was stored at $4^{\circ} \mathrm{C}$ for subsequent experiments.

\section{Preparation of Standard Substance Stock Solution}

Eleven standard substances (geniposidic acid, neochlorogenic acid, chlorogenic acid, caffeic acid, geniposide, genipin, pinoresinol di-o-glucopyranoside, syringaresinol di-oglucopyranoside, isochlorogenic acid A, pinoresinol $o$ glucopyranoside, and isochlorogenic acid C) were accurately weighed and respectively dissolved with methanol solvent. The separate standard solutions were then mixed as stock solution for plotting standard curves through stepwise dilution.

\section{UHPLC-Q-TOF/MS Acquisition Analysis}

UHPLC-Q-TOF/MS system was composed of Agilent 1290 UHPLC instrument (Agilent Technologies, Waldbronn, Germany) and Agilent 6520 Q-TOF mass spectrometer (Agilent Corporation, Santa Clara, CA, USA). The mass spectra data was acquired in the negative electrospray ion (ESI) mode. The chromatographic peaks were separated on an ACQUITY UPLC BEH C ${ }_{18}$ Column $(2.1 \times 150 \mathrm{~mm}, 1.7 \mu \mathrm{m}$, Waters) at a flow rate of $0.3 \mathrm{ml} / \mathrm{min}$. The temperature of column was at room temperature $\left(28^{\circ} \mathrm{C}\right)$. Mobile phase consisted of $0.1 \%$ formic acid-water (A) and acetonitrile (B). The gradient elution program was set as: $0-2.5 \mathrm{~min}, 5 \%-10 \% \mathrm{~B} ; 2.5-7 \mathrm{~min}, 10 \%-13 \%$ B; 7-10 min, $13 \%-15 \% \mathrm{~B}$; $10-11 \mathrm{~min}, 15 \%-18 \% \mathrm{~B} ; 11-15 \mathrm{~min}$, $18 \%-30 \% \mathrm{~B} ; 15-17 \mathrm{~min}, 30 \%-45 \% \mathrm{~B} ; 17-22 \mathrm{~min}, 45 \%-95 \% \mathrm{~B}$; 22-27 $\mathrm{min}, 95 \%-5 \% \mathrm{~B}$. The post run time was $5 \mathrm{~min}$. The injection was $5 \mu \mathrm{l}$. The related Q-TOF/MS parameters were listed as follows: drying gas, $\mathrm{N}_{2}$; gas flow rate, $11 \mathrm{~L} / \mathrm{min}$; drying gas temperature, $330^{\circ} \mathrm{C}$; nebulizer gas pressure, 40 psig; capillary voltage, $3500 \mathrm{~V}$; fragmentor voltage, $120 \mathrm{~V}$; skimmer voltage, 65 $\mathrm{V}$; octopole RF, $750 \mathrm{~V}$; collision energy (CE), 20 and $30 \mathrm{~V}$. The scan range of mass spectra was m/z $100-1,500$.

\section{UHPLC-PDA Analysis}

The quantitative analysis was carried out by the Waters Acquity UHPLC instrument (Waters Corp., Milford, MA, USA) using with PDA. The chromatography column, mobile phase and flow rate setting were as same as UPLC-Q-TOF/MS method. The column temperature was $40^{\circ} \mathrm{C}$. The gradient elution program was set as: $0-2.5 \mathrm{~min}, 5 \%-10 \% \mathrm{~B} ; 2.5-5.5 \mathrm{~min}, 10 \%-11 \% \mathrm{~B}$; $5.5-6$ $\min , 11 \%-12 \%$ B; 6-10 min, $12 \%-15 \%$ B; $10-13 \min , 15 \%-17 \%$ B; $13-14 \mathrm{~min}, 17 \%-25 \% \mathrm{~B}$; $14-15 \mathrm{~min}, 25 \%-5 \% \mathrm{~B}$. The post run was $3 \mathrm{~min}$. The optimal absorbed wavelengths were respectively $240 \mathrm{~nm}$ for geniposidic acid, geniposide, genipin, and pinoresinol di-o-glucopyranoside; $227 \mathrm{~nm}$ for syringaresinol di-oglucopyranoside; and $327 \mathrm{~nm}$ for neochlorogenic acid, chlorogenic acid, caffeic acid, isochlorogenic acid A, and isochlorogenic acid C. In order to make the quantitative analysis more convenient, the multiwavelengths switch method was employed and was set as follows: $1.20-2.15 \mathrm{~min}, 240-327$ $\mathrm{nm}$; 2.15-4.50 $\min , 327-240 \mathrm{~nm}$; 4.50-7.15 min, 240-227 nm; 7.15-10 min, 227-327; 10-12.24 min, 327-227 nm; 12.24-13.00 $\mathrm{min}, 227-327 \mathrm{~nm}$. The injection volume was $3 \mu \mathrm{l}$.

\section{Qualitative Analysis Method}

Six types of compounds in Eucommiae Cortex have been summarized on the basis of the literatures, including lignans, phenylpropanoids, iridoids, phenolic acids, and others. The chemical formula and name of all the compounds collected were imported into an excel file and saved as the.csv form. Then the in-house compounds library of Eucommiae Cortex had been completed and was used to quickly found out the compounds of interest from the massive raw $\mathrm{MS}^{2}$ data by the function "find by formula" on the Agilent MassHunter Qualitative Workstation Analysis B.07.00 (Agilent Technologies Inc., Santa Clara, CA, USA). At last, the in-depth identification was performed by matching real MS, $\mathrm{MS}^{2}$ data from the EIC (extraction ion chromatography) with the related information in the literatures, especially, the characteristic ions and fragment pattern.

\section{Method Validation}

\section{UHPLC-Q-TOF/MS Acquisition Method Validation}

The precision, repeatability, and stability were investigated to validate the applicability of UHPLC-Q-TOF/MS method by using the QC samples. All sample solutions were mixed in a certain volume to prepare for the QC sample. The six independent QC samples were subject to UHPLC-Q-TOF/MS analysis within one day and three continuous days for evaluating the precision. The same QC sample was injected six times to assess the repeatability of acquisition method. The stability was conducted by analyzing response intensity of the target analytes in the QC samples at $0,2,4,6,8,12$, and $24 \mathrm{~h}$. All the above validation results were presented as the relative standard deviation (RSD).

\section{UHPLC-PDA Quantitative Method Validation}

The mixed standard stock solution was stepwise diluted into the different working concentrations required by each calibration curve. Calibration curves required was plotted with the peak area as $\mathrm{X}$-axis and the concentrations of target compounds as $\mathrm{Y}$-axis, respectively. The mixed standard solution containing 11 analytes was gradually diluted into the concentrations where the ratio of signal to noise $(S / N)$ was detected as 3 and 10 , respectively. These mixed standard solutions were then used to evaluate the limits of 
detection (LOD) and limits of quantification (LOQ). The precision and accuracy of intra- and interday were analyzed by calculating the RSD values of the mixed standard solutions with three different concentrations (low, medium, and high). The repeatability of UHPLC-PDA quantitative method was evaluated by extraction and analysis of the target compounds in six independent samples. The sample solutions were repeatedly injected six times to explore stability at $0,2,4,6,8,10,12$, and $24 \mathrm{~h}$ in room temperature $\left(28^{\circ} \mathrm{C}\right)$, respectively. The recovery experiment was conducted by adding the certain quantity of 11 standards mixture to the samples and the results were assessed by recovery rate $(\%)$.

\section{Data Analysis}

Firstly, all the raw data acquired in (-)-ESI mode were introduced to the $\mathrm{R}$ software ( $\mathrm{R}$ Foundation for Statistical Computing, Vienna, Austria) where all $\mathrm{mz}$ values detected would be normalized. Secondly, a mass of above metabolomics data was used for the orthogonal partial least-squares discriminant analysis (OPLS-DA) by the Simca-P (version 14.1, Umetrics, Umea, Sweden) in order to initially filter the candidate compounds. Thirdly, the accuracy of CdQMs was validated by PLSR and RF algorithms on Matlab R2015B (Mathworks, Natick, USA). At last, the discriminant function was used to evaluate the applicability of filtered CdQM and predict the types of unknown Eucommiae Cortex products by SPSS 17.0 (SPSS, Chicago, IL, USA).

\section{RESULTS AND DISCUSSION}

\section{UHPLC-Q-TOF/MS Acquisition Method Validation}

The retention times (Rt), mass to charge ratios $(\mathrm{m} / \mathrm{z})$, and peak areas of 11 CdQMs were employed to calculate the RSD values, which were regarded as the important assessment indicator of precision, repeatability, and stability. It was acceptable that the RSD values were no more than $5 \%$. The RSD values of intra- and interday precisions were all below $3.46 \%$, which displayed a high accuracy of Rt, $\mathrm{m} / \mathrm{z}$, and peak areas of target ions in the process of multiple samples analysis by the UHPLC-Q-TOF/MS method. Moreover, the repeatability with the RSDs ranging from $0.00 \%$ to $3.86 \%$ showed good consistency of results detected by UHPLCQ-TOF/MS. Finally, the RSDs indicative of stability were within $0.00 \%-3.30 \%$, demonstrating that sample solutions was enough stable for qualitative detection in $24 \mathrm{~h}$. In conclusion, all the above results (Table S1) indicated that this UHPLC-Q-TOF/MS method was applicable and reliable for acquiring the metabolomics data.

\section{Compound Identification in Crude Eucommiae Cortex}

\section{Acquisition of Chemical Compounds Information}

The identification of chemical compounds was essential for filtering the candidate markers in the following study. The whole chemical profile of Eucommiae Cortex was acquired in the negative ESI mode. In general, the qualitative analysis was time-consuming and labor-intensive due to the massive $\mathrm{MS}^{1}$ and
$\mathrm{MS}^{2}$ data. However, we built the in-house library for the targeted identification, which could rapidly search the known compounds from complex mass spectra data. A total of 72 candidate compounds (Table S2) were initially extracted from the MS/ MS spectra data. The same compound might hit for several times, whereas the hitting peaks appeared at different retention times. These peaks possibly represented isomers. Therefore, 72 candidate compounds need to be further identified by matching the accuracy MS data (error $<5$ ppm), key characteristic ions, and chromatographic elution order with that in the literatures to exclude the false positive results. Finally, 67 compounds (Table 1 and Figure 1) in Eucommiae Cortex were tentatively identified, containing 31 lignans, 10 iridoids, 10 phenylpropanoids, 6 organic acids, 10 other compounds.

\section{Identification of Lignans}

Lignans and their derivatives were a main class of secondary metabolites in Eucommiae Cortex, and display various bioactivities in vivo or in vitro (Deyama, 1983; Shi et al., 2013). In this work, 31 lignans have been tentatively characterized, including compounds 16, 24-25, 28-30, 34-37, 42-47, 49-52, 54, 55, 57-65 (Brenes et al., 2000; Guo et al., 2007; Feng et al., 2007; Chai et al., 2012; Pi et al., 2016; He et al., 2018; Jia et al., 2019; Jiang et al., 2019; Qi et al., 2019). The most lignans in Eucommiae Cortex are phenylpropanoid dimers with one or two glucose units, which means a few of the $\mathrm{MS}^{2}$ fragments followed by the loss of glucose neutral moiety. Moreover, the $\mathrm{MS}^{2}$ spectrum of lignans showed several key characteristic ions at $\mathrm{m} / \mathrm{z} 327,311$, 181 , and 150 , which were mainly attributed to cleavage of the tetrahydrofuran ring and losses of $\mathrm{CH}_{3}, \mathrm{CH}_{2} \mathrm{O}, \mathrm{CO}, \mathrm{CH}_{3} \mathrm{O}$, and $\mathrm{CH}_{3} \mathrm{OH}$ (Guo et al., 2007; Jiang et al., 2019). Take several compounds for examples to illustrate the qualitative process. The quasi-molecular ion $[\mathrm{M}-\mathrm{H}]^{-}$of compound 29 at $\mathrm{m} / \mathrm{z} 681$ corresponded to the formula $\mathrm{C}_{32} \mathrm{H}_{42} \mathrm{O}_{16}$. Its $\mathrm{MS}^{2}$ fragmental ions at $\mathrm{m} / \mathrm{z} 519$ and $\mathrm{m} / \mathrm{z} 357$ were observed due to the loss of 1 and 2 glucose groups, respectively, and $\mathrm{MS}^{2}$ ion at $\mathrm{m} / \mathrm{z} 151$ was generated by the cleavage of tetrahydrofuran-ring. The compound 29 was thereof identified as pinoresinol di-oglucopyranoside (Brenes et al., 2000; Feng et al., 2007). The parent ion $\left[\mathrm{C}_{20} \mathrm{H}_{22} \mathrm{O}_{6}-\mathrm{H}\right]^{-}$of compound 45 at m/z 357 firstly was converted into the characteristic ion at $\mathrm{m} / \mathrm{z} 327$ due to the cleavage of tetrahydrofuran-ring. Moreover, another characteristic ion at $\mathrm{m} / \mathrm{z} 311$ was also observed owing to the loss of $\mathrm{CH}_{3}(15 \mathrm{Da})$ from the ion at $\mathrm{m} / \mathrm{z}$ 327. Thus, the compound 45 was tentatively identified as pinoresinol (Brenes et al., 2000). As to the compound 46, its parent ion $\left[\mathrm{C}_{26} \mathrm{H}_{32} \mathrm{O}_{11^{-}}\right.$ $\mathrm{H}]^{-}$at $\mathrm{m} / \mathrm{z} 591$ was lower $162 \mathrm{Da}$ than that of compound 29. Additionally, it shared the similar characteristic ions to compound 29 at $\mathrm{m} / \mathrm{z} 311,297$. Finally, compound 46 was rapidly identified as pinoresinol-o-glucopyranoside (Qi et al., 2019). The compounds 42 and 52 with $[\mathrm{M}-\mathrm{H}]^{-}$ion at $\mathrm{m} / \mathrm{z} 373$ had another characteristic ion at $\mathrm{m} / \mathrm{z} 165$ and further produced ion with $\mathrm{m} / \mathrm{z} 150$ by the loss of $\mathrm{CH}_{3}$. Compared the real retention behavior with that in the reported literatures (He et al., 2018), compounds $\mathbf{4 2}$ and $\mathbf{5 2}$ were tentatively identified as erythroguaiacylglycerol- $\beta$-conifery aldehyde ether and threoguaiacylglycerol- $\beta$-conifery aldehyde ether, respectively. 
TABLE 1 | The identification of constituents of crude Eucommiae Cortex extract by UHPLC-Q-TOF/MS in negative ion mode.

\begin{tabular}{|c|c|c|c|c|c|c|c|c|c|}
\hline $\begin{array}{l}\text { Cpd } \\
\text { no. }\end{array}$ & $\begin{array}{c}\mathbf{R t} \\
(\min )\end{array}$ & Formula & {$[\mathrm{M}-\mathrm{H}]-$} & $\begin{array}{c}{[\mathrm{M}} \\
+\mathrm{COOH}]-\end{array}$ & MS/MS(-) & $\Delta \mathrm{ppm}$ & Identification & & References \\
\hline 1 & 1.178 & $\mathrm{C}_{6} \mathrm{H}_{8} \mathrm{O}_{7}$ & 191.0196 & & $\begin{array}{l}111.0076, \\
129.0178, \\
154.9993, \\
173.0085\end{array}$ & 3.26 & Isocitric acid & Others & $\begin{array}{l}\text { He et al., } \\
2018 \text {; Lei et al., } \\
2018\end{array}$ \\
\hline 2 & 1.45 & $\mathrm{C}_{15} \mathrm{H}_{22} \mathrm{O}_{9}$ & & 391.1231 & $\begin{array}{l}101.0243, \\
111.0064, \\
147.0446, \\
165.0551 \\
183.0652\end{array}$ & 4.29 & Aucubin & Iridoids & $\begin{array}{l}\text { Allen et al., } \\
\text { 2015; He et al., } \\
\text { 2018; Jiang et al, } \\
2019\end{array}$ \\
\hline 3 & 1.534 & $\mathrm{C}_{16} \mathrm{H}_{22} \mathrm{O}_{11}$ & 389.1090 & & $\begin{array}{c}113.0245, \\
119.0368, \\
139.0394 \\
147.0427 \\
165.0552 \\
183.0658 \\
209.0448 \\
227.0539\end{array}$ & 0.47 & Deacetylasperulosidic acid & Iridoids & He et al., 2018 \\
\hline 4 & 2.077 & $\mathrm{C}_{13} \mathrm{H}_{16} \mathrm{O}_{9}$ & 315.0714 & & $\begin{array}{l}108.0195 \\
153.0159\end{array}$ & 2.39 & Protocatechuicacid-4-glucoside & Phenylpropanoids & $\begin{array}{l}\text { He et al., } \\
2018\end{array}$ \\
\hline 5 & 2.194 & $\mathrm{C}_{15} \mathrm{H}_{20} \mathrm{O}_{12} \mathrm{~S}$ & 423.0598 & & $\begin{array}{c}119.0497 \\
163.0396 \\
199.0068 \\
215.0008 \\
242.9988 \\
261.008 \\
303.1024\end{array}$ & 1.11 & $\begin{array}{l}\text { 6-(4-Formyl-2,6- dimethoxyphenoxy)-3,4,5- } \\
\text { trihydroxytetrahydro-2H-pyran-2-yl) methyl } \\
\text { hydrogen sulfate }\end{array}$ & Others & $\begin{array}{l}\text { He et al., } \\
2018\end{array}$ \\
\hline 6 & 2.212 & $\mathrm{C}_{14} \mathrm{H}_{18} \mathrm{O}_{9}$ & 329.0873 & & $\begin{array}{c}123.0443 \\
152.011 \\
167.0342\end{array}$ & 1.53 & 2-Glucopyranosyloxy-5- hydroxyphenyl acetic acid & Organic acids & $\begin{array}{l}\text { He et al., } \\
2018\end{array}$ \\
\hline 7 & 2.28 & $\mathrm{C}_{8} \mathrm{H}_{8} \mathrm{O}_{4}$ & 167.0341 & & $\begin{array}{c}108.02303 \\
123.0413 \\
152.0104\end{array}$ & 0.80 & Vanillic acid & Organic acids & $\begin{array}{l}\text { Lei et al., } \\
2018\end{array}$ \\
\hline 8 & 2.348 & $\mathrm{C}_{16} \mathrm{H}_{22} \mathrm{O}_{10}$ & 373.1125 & & $\begin{array}{l}\text { 101.0242, } \\
\text { 123.0447, } \\
\text { 147.0431, } \\
\text { 149.0602, } \\
\text { 167.0704, } \\
193.0497 \\
211.0606\end{array}$ & 3.97 & Geniposidic acid & Iridoids & $\begin{array}{l}\text { Allen et al., } \\
\text { 2015; He et al., } \\
\text { 2018; Jiang et al., } \\
2019\end{array}$ \\
\hline 9 & 2.534 & $\mathrm{C}_{7} \mathrm{H}_{6} \mathrm{O}_{4}$ & 153.0186 & & 109.0293 & 4.78 & 3,4-Dihydroxy benzoic acid & Organic acids & $\begin{array}{l}\text { He et al., } \\
2018 \text {; Jia et al., } \\
2019\end{array}$ \\
\hline 10 & 2.753 & $\mathrm{C}_{15} \mathrm{H}_{20} \mathrm{O}_{10}$ & 359.0981 & & $\begin{array}{c}123.0087 \\
138.032 \\
153.05331 \\
166.9983 \\
182.0214 \\
197.0444\end{array}$ & 0.75 & $\begin{array}{l}\text { 4-Glucopyranosyloxy-3,5- } \\
\text { dimethoxy benzoic acid }\end{array}$ & Organic acids & $\begin{array}{l}\text { He et al., } \\
2018 \text {; Jia et al., } \\
2019\end{array}$ \\
\hline 11 & 2.874 & $\mathrm{C}_{16} \mathrm{H}_{18} \mathrm{O}_{9}$ & 353.0883 & & $\begin{array}{c}135.0451 \\
161.025 \\
173.0453 \\
179.0341 \\
191.0557\end{array}$ & -1.40 & Neochlorogenic acid & Phenylpropanoids & $\begin{array}{l}\text { Allen et al., } \\
2015 \text {; He et al., } \\
2018\end{array}$ \\
\hline 12 & 3.008 & $\mathrm{C}_{15} \mathrm{H}_{24} \mathrm{O}_{10}$ & 363.1281 & & $\begin{array}{l}101.0246, \\
105.0191 \\
123.0445 \\
147.0295\end{array}$ & 4.31 & Harpagide & Iridoids & $\begin{array}{l}\text { He et al., } \\
2018\end{array}$ \\
\hline 13 & 3.484 & $\mathrm{C}_{13} \mathrm{H}_{24} \mathrm{O}_{9}$ & 323.1336 & & $\begin{array}{l}101.0235 \\
113.0237 \\
119.0344, \\
131.0352, \\
143.0386\end{array}$ & 3.63 & Periplobiose & Others & $\begin{array}{l}\text { He et al., } \\
2018 \text {; Jia et al., } \\
2019\end{array}$ \\
\hline
\end{tabular}


TABLE 1 | Continued

\begin{tabular}{|c|c|c|c|c|c|c|c|c|c|}
\hline $\begin{array}{l}\text { Cpd } \\
\text { no. }\end{array}$ & $\begin{array}{c}\mathbf{R t} \\
(\min )\end{array}$ & Formula & {$[\mathrm{M}-\mathrm{H}]-$} & $\begin{array}{c}{[\mathrm{M}} \\
+\mathrm{COOH}]-\end{array}$ & MS/MS(-) & $\Delta$ ppm & Identification & & References \\
\hline 14 & 3.568 & $\mathrm{C}_{7} \mathrm{H}_{6} \mathrm{O}_{3}$ & 137.0241 & & $\begin{array}{l}108.0218, \\
109.0282, \\
119.0153 \\
136.0158\end{array}$ & 2.30 & 3-Hydroxybenzoic acid & Others & $\begin{array}{l}\text { He et al., } \\
2018 \text {; Jia et al., } \\
2019\end{array}$ \\
\hline 15 & 3.702 & $\mathrm{C}_{22} \mathrm{H}_{28} \mathrm{O}_{14}$ & 515.1398 & & $\begin{array}{l}179.0351 \\
191.0555 \\
323.0786\end{array}$ & 1.61 & cis 5-o-(3' -o-caffeoyl glucosyl) quinic acid & Phenylpropanoids & $\begin{array}{l}\text { He et al., } \\
2018 \text {; Jia et al., } \\
2019\end{array}$ \\
\hline 16 & 4.109 & $\mathrm{C}_{32} \mathrm{H}_{44} \mathrm{O}_{17}$ & & 745.2532 & $\begin{array}{l}179.0706 \\
195.0657 \\
327.1237 \\
345.1349 \\
357.1306 \\
375.1444 \\
537.1926\end{array}$ & 4.07 & Olivil 4',4"-di-o-glucopyranoside & Lignans & $\begin{array}{l}\text { He et al., } \\
\text { 2018; Jiang et al., } \\
2019\end{array}$ \\
\hline 17 & 4.16 & $\mathrm{C}_{16} \mathrm{H}_{18} \mathrm{O}_{9}$ & 353.0883 & & $\begin{array}{l}135.0446 \\
155.0341 \\
161.0239 \\
173.0448 \\
179.0344 \\
191.0559\end{array}$ & -1.40 & Chlorogenic acid & Phenylpropanoids & $\begin{array}{l}\text { Allen et al., } \\
\text { 2015; He et al., } \\
\text { 2018; Jia et al., } \\
2019\end{array}$ \\
\hline 18 & 4.492 & $\mathrm{C}_{16} \mathrm{H}_{18} \mathrm{O}_{9}$ & 353.0876 & & $\begin{array}{l}135.0440 \\
155.0351 \\
161.0225 \\
173.0444 \\
179.0340 \\
191.0554\end{array}$ & 0.58 & Cryptochlorogenic acid & Phenylpropanoids & $\begin{array}{l}\text { Allen et al., } \\
2015 \text {; He et al., } \\
2018\end{array}$ \\
\hline 19 & 4.636 & $\mathrm{C}_{9} \mathrm{H}_{8} \mathrm{O}_{4}$ & 179.0355 & & $\begin{array}{l}109.0308, \\
117.0338, \\
134.0364, \\
135.0446\end{array}$ & -2.88 & Caffeic acid & Phenylpropanoids & $\begin{array}{l}\text { Zhang et al., } \\
\text { 2016; He et al., } \\
2018\end{array}$ \\
\hline 20 & 5.132 & $\mathrm{C}_{17} \mathrm{H}_{22} \mathrm{O}_{10}$ & 385.1136 & & $\begin{array}{l}101.0239 \\
123.0465 \\
177.0550\end{array}$ & 1.09 & $\begin{array}{l}\text { 4-[3-Glucopyranosyloxy-2- hydroxyphenyl]-3- } \\
\text { methyl-4- oxobutanoic acid }\end{array}$ & Organic acids & $\begin{array}{l}\text { He et al., } \\
2018\end{array}$ \\
\hline 21 & 5.465 & $\mathrm{C}_{18} \mathrm{H}_{26} \mathrm{O}_{10}$ & & 447.1501 & $\begin{array}{l}101.0242 \\
111.0083 \\
134.0326 \\
149.0487 \\
161.0453 \\
233.0655 \\
251.0941 \\
269.1011\end{array}$ & 1.99 & 4-[2-(Xylopyranosyloxy)ethyl] phenylxylopyranoside & Others & $\begin{array}{l}\text { He et al., } \\
2018\end{array}$ \\
\hline 22 & 5.742 & $\mathrm{C}_{16} \mathrm{H}_{18} \mathrm{O}_{8}$ & 337.0933 & & $\begin{array}{c}161.0239 \\
163.0398 \\
191.0555\end{array}$ & -1.21 & 3-p-coumaroylquinic acid & Phenylpropanoids & $\begin{array}{l}\text { He et al., } \\
2018\end{array}$ \\
\hline 23 & 5.929 & $\mathrm{C}_{17} \mathrm{H}_{24} \mathrm{O}_{10}$ & & 433.1341 & $\begin{array}{l}101.0244 \\
105.0323 \\
123.0450 \\
147.0401 \\
207.0657 \\
225.0768\end{array}$ & -2.70 & Geniposide & Iridoids & $\begin{array}{l}\text { He et al., } \\
2018 \text {; Jia et al., } \\
2019\end{array}$ \\
\hline 24 & 6.07 & $\mathrm{C}_{26} \mathrm{H}_{34} \mathrm{O}_{12}$ & 537.1956 & & $\begin{array}{l}\text { 151.0352, } \\
297.1106 \\
312.1036 \\
327.1236 \\
345.1367 \\
357.1324\end{array}$ & 3.99 & Olivil 4' -o-glucopyranoside & Lignans & $\begin{array}{l}\text { He et al., 2018; } \\
\text { Jiang et al., } \\
2019\end{array}$ \\
\hline 25 & 6.552 & $\mathrm{C}_{32} \mathrm{H}_{42} \mathrm{O}_{17}$ & 697.2336 & & $\begin{array}{l}373.1291 \\
535.1812\end{array}$ & 1.90 & I-Hydroxypinoresinol di-o-glucopyranoside & Lignans & $\begin{array}{l}\text { He et al., } \\
2018\end{array}$ \\
\hline 26 & 6.62 & $\mathrm{C}_{33} \mathrm{H}_{44} \mathrm{O}_{19}$ & 743.2380 & & $\begin{array}{l}343.1179 \\
373.1286 \\
535.1886\end{array}$ & 3.23 & Naringin DHC 4-o- $\beta$-d- glucopyranoside & Others & $\begin{array}{l}\text { He et al., } \\
\text { 2018; Jia et al., } \\
2019\end{array}$ \\
\hline
\end{tabular}


TABLE 1 | Continued

\begin{tabular}{|c|c|c|c|c|c|c|c|c|c|}
\hline $\begin{array}{l}\text { Cpd } \\
\text { no. }\end{array}$ & $\begin{array}{c}\text { Rt } \\
(\min )\end{array}$ & Formula & [M-H]- & $\begin{array}{c}{[\mathrm{M}} \\
+\mathrm{COOH}]-\end{array}$ & MS/MS(-) & $\Delta \mathrm{ppm}$ & Identification & & References \\
\hline 27 & 7.62 & $\mathrm{C}_{11} \mathrm{H}_{14} \mathrm{O}_{5}$ & 225.0767 & & $\begin{array}{l}101.0244, \\
105.0347, \\
119.0500, \\
123.0445, \\
147.0444, \\
207.0663\end{array}$ & 0.65 & Genipin & Iridoids & $\begin{array}{l}\text { Allen et al., } \\
\text { 2015; Zhang } \\
\text { et al., } \\
\text { 2016; He et al., } \\
2018\end{array}$ \\
\hline 28 & 7.907 & $\mathrm{C}_{26} \mathrm{H}_{34} \mathrm{O}_{12}$ & 537.1956 & & $\begin{array}{l}151.0354 \\
195.0659 \\
297.1106 \\
327.1200 \\
375.1403\end{array}$ & 3.99 & Olivil 4"-o-glucopyranoside & Lignans & He et al., 2018 \\
\hline 29 & 8.162 & $\mathrm{C}_{32} \mathrm{H}_{42} \mathrm{O}_{16}$ & 681.2373 & & $\begin{array}{c}136.0165 \\
151.0395 \\
175.076 \\
327.1279 \\
342.1108 \\
357.1350 \\
519.1824\end{array}$ & 3.97 & Pinoresinol di-o-glucopyranoside & Lignans & $\begin{array}{l}\text { Brenes et al., } \\
2000 \text {; Feng et al., } \\
2007\end{array}$ \\
\hline 30 & 8.705 & $\mathrm{C}_{32} \mathrm{H}_{42} \mathrm{O}_{16}$ & 681.2376 & & $\begin{array}{c}179.0536 \\
339.1248 \\
01.1789 \\
219.1864\end{array}$ & 3.97 & Dehydrodiconiferyl 4, $\gamma$ '-o-glucopyranoside & Lignans & $\begin{array}{l}\text { He et al., } \\
2018\end{array}$ \\
\hline 31 & 8.974 & $\mathrm{C}_{17} \mathrm{H}_{20} \mathrm{O}_{9}$ & 367.1028 & & $\begin{array}{l}134.0366, \\
173.0422, \\
191.0551\end{array}$ & 1.78 & 5-o-feruloylquinic acid & Phenylpropanoids & $\begin{array}{l}\text { He et al., } \\
\text { 2018; Jia et al., } \\
2019\end{array}$ \\
\hline 32 & 9.112 & $\mathrm{C}_{23} \mathrm{H}_{26} \mathrm{O}_{13}$ & 509.1295 & & $\begin{array}{c}123.0442 \\
153.0181 \\
182.0212 \\
197.0451 \\
211.0608 \\
297.0624 \\
311.0764\end{array}$ & 1.11 & $\begin{array}{l}\text { 4,8,9,10-tetrahydroxy-3,6,7-trimethoxy-2-anthryl- } \\
\text { glucopyranoside }\end{array}$ & Organic acids & $\begin{array}{l}\text { He et al., } \\
\text { 2018; Jia et al., } \\
2019\end{array}$ \\
\hline 33 & 9.196 & $\mathrm{C}_{33} \mathrm{H}_{46} \mathrm{O}_{18}$ & 729.2584 & & $\begin{array}{l}165.0553 \\
491.1922 \\
503.1920 \\
521.2033\end{array}$ & 3.75 & $\begin{array}{l}\text { 3-[4-(2-[4-Glucopyranosyloxy- } \\
\text { 3-methoxyphenyl)-2-hydroxy- } \\
\text { 1-(hydroxymethyl) ethoxy]- } \\
\text { 3,5-dimethoxyphenyl]-2- propen-1- } \\
\text { ylglucopyranoside }\end{array}$ & Others & $\begin{array}{l}\text { He et al., } \\
2018\end{array}$ \\
\hline 34 & 9.315 & $\mathrm{C}_{33} \mathrm{H}_{44} \mathrm{O}_{17}$ & 711.2487 & & $\begin{array}{l}387.1447 \\
549.1960\end{array}$ & 2.63 & Medioresinol di-o-glucopyranoside & Lignans & $\begin{array}{l}\text { He et al., } \\
2018\end{array}$ \\
\hline 35 & 9.738 & $\mathrm{C}_{26} \mathrm{H}_{32} \mathrm{O}_{12}$ & 535.1800 & & $\begin{array}{c}181.0499 \\
269.0827 \\
298.082 \\
313.1096 \\
325.1005 \\
343.1183 \\
358.1068\end{array}$ & 3.92 & I-Hydroxypinoresinol 4'-o-glucopyranoside & Lignans & $\begin{array}{l}\text { He et al., 2018; } \\
\text { Jiang et al., } \\
2019\end{array}$ \\
\hline 36 & 10.196 & $\mathrm{C}_{26} \mathrm{H}_{32} \mathrm{O}_{12}$ & 535.1800 & & $\begin{array}{l}151.0419, \\
181.0494, \\
298.0839, \\
313.1062, \\
343.1171 \\
373.1294\end{array}$ & 3.92 & I-Hydroxypinoresinol 4"-o-glucopyranoside & Lignans & $\begin{array}{l}\text { He et al., 2018; } \\
\text { Jiang et al., } \\
2019\end{array}$ \\
\hline 37 & 10.281 & $\mathrm{C}_{34} \mathrm{H}_{46} \mathrm{O}_{18}$ & 741.2593 & & $\begin{array}{l}166.0294, \\
181.0491, \\
357.1347 \\
371.1440 \\
402.1312, \\
417.1552\end{array}$ & 2.48 & Syringaresinol di-o-glucopyranoside & Lignans & $\begin{array}{l}\text { Chai et al., } \\
\text { 2012; He et al., } \\
2018\end{array}$ \\
\hline 38 & 10.671 & $\mathrm{C}_{10} \mathrm{H}_{1} \mathrm{O}_{5}$ & 217.1078 & & $\begin{array}{l}123.0758 \\
127.0724 \\
137.0953 \\
155.1075\end{array}$ & 1.59 & Epieucommiol & Iridoids & $\begin{array}{l}\text { He et al., } \\
\text { 2018; Jiang et al., } \\
\text { 2019; Jia et al., } \\
2019\end{array}$ \\
\hline
\end{tabular}


TABLE 1 | Continued

\begin{tabular}{|c|c|c|c|c|c|c|c|c|c|}
\hline $\begin{array}{l}\text { Cpd } \\
\text { no. }\end{array}$ & $\begin{array}{c}\text { Rt } \\
(\min )\end{array}$ & Formula & [M-H]- & $\stackrel{[\mathrm{M}}{+\mathrm{COOH}]-}$ & MS/MS(-) & $\Delta$ ppm & Identification & & References \\
\hline & & & & & $\begin{array}{l}171.1015 \\
199.0947\end{array}$ & & & & \\
\hline 39 & 11.838 & $\mathrm{C}_{23} \mathrm{H}_{26} \mathrm{O}_{13}$ & 509.1292 & & $\begin{array}{c}123.0445 \\
152.0115 \\
167.0340 \\
183.0297 \\
197.0455 \\
327.0710\end{array}$ & 1.69 & $\begin{array}{l}\text { 4-\{[6-o-(4-hydroxy-3,5- dimethoxybenzoyl)- } \\
\text { glucopyranosyl]oxy\}-3- methoxybenzoic acid }\end{array}$ & Others & He et al., 2018 \\
\hline 40 & 12.094 & $\mathrm{C}_{25} \mathrm{H}_{31} \mathrm{NO}_{11}$ & 520.1811 & & $\begin{array}{l}101.0248 \\
147.0446\end{array}$ & 2.56 & Eucomoside B & Iridoids & $\begin{array}{l}\text { Allen et al., } \\
\text { 2015; He et al., } \\
2018\end{array}$ \\
\hline 41 & 12.705 & $\mathrm{C}_{25} \mathrm{H}_{24} \mathrm{O}_{12}$ & 515.1189 & & $\begin{array}{l}135.0444 \\
155.0355 \\
161.0245 \\
173.0450 \\
179.0346 \\
191.0552 \\
335.0780 \\
353.0865\end{array}$ & 1.16 & Isochlorogenic acid A & Phenylpropanoids & $\begin{array}{l}\text { He et al., } \\
2018 \text {; Jia et al., } \\
2019\end{array}$ \\
\hline 42 & 12.774 & $\mathrm{C}_{20} \mathrm{H}_{22} \mathrm{O}_{7}$ & 373.1291 & & $\begin{array}{l}150.0317 \\
162.0331 \\
165.0543 \\
177.0525 \\
180.0418\end{array}$ & 0.47 & Erythro-guaiacylglycerol- $\beta$ - conifery aldehyde ether & Lignans & He et al., 2018 \\
\hline 43 & 12.79 & $\mathrm{C}_{43} \mathrm{H}_{56} \mathrm{O}_{21}$ & 907.3220 & & $\begin{array}{l}165.0550 \\
195.0678 \\
357.1308 \\
387.1445 \\
535.1994 \\
565.2034 \\
745.2721 \\
861.2972\end{array}$ & 2.35 & Hedyotol C di-o- glucopyranoside & Lignans & $\begin{array}{l}\text { He et al., } \\
\text { 2018; Jiang et al., } \\
2019\end{array}$ \\
\hline 44 & 12.858 & $\mathrm{C}_{43} \mathrm{H}_{54} \mathrm{O}_{22}$ & 921.3000 & & $\begin{array}{l}341.0882 \\
417.1579 \\
759.2532\end{array}$ & 3.68 & Unknown & Lignans & He et al., 2018 \\
\hline 45 & 12.991 & $\mathrm{C}_{20} \mathrm{H}_{22} \mathrm{O}_{6}$ & 357.1342 & & $\begin{array}{l}136.0155 \\
175.0727 \\
297.1164 \\
311.1266 \\
327.0943\end{array}$ & 0.45 & Pinoresinol & Lignans & $\begin{array}{l}\text { Brenes et al., } \\
2000\end{array}$ \\
\hline 46 & 12.994 & $\mathrm{C}_{26} \mathrm{H}_{32} \mathrm{O}_{11}$ & 519.1862 & & $\begin{array}{l}136.0160 \\
151.0396 \\
175.0754 \\
297.1131 \\
311.1287 \\
357.1348\end{array}$ & 1.89 & Pinoresinol-o-glucopyranoside & Lignans & $\begin{array}{l}\text { Qi et al., } \\
2019\end{array}$ \\
\hline 47 & 13.059 & $\mathrm{C}_{27} \mathrm{H}_{34} \mathrm{O}_{12}$ & 549.1978 & & $\begin{array}{l}136.0154, \\
166.0269, \\
181.0505 \\
372.1212 \\
387.1451\end{array}$ & -0.45 & Medioresinol 4"-o- $\beta$-d- glucopyranoside & Lignans & $\begin{array}{l}\text { He et al., } \\
\text { 2018; Jiang et al., } \\
2019\end{array}$ \\
\hline 48 & 13.113 & $\mathrm{C}_{9} \mathrm{H}_{16} \mathrm{O}_{4}$ & 187.0972 & & $\begin{array}{l}\text { 123.0813, } \\
143.1073 \\
169.0864\end{array}$ & 2.03 & Eucommiol & Iridoids & $\begin{array}{l}\text { He et al., } \\
\text { 2018; Jiang et al., } \\
\text { 2019; Jia et al., } \\
2019\end{array}$ \\
\hline 49 & 13.333 & $\mathrm{C}_{44} \mathrm{H}_{58} \mathrm{O}_{22}$ & 937.3316 & & $\begin{array}{l}387.1405 \\
891.2881\end{array}$ & 3.30 & $\begin{array}{l}\text { Glycerol-syringaresinol ether } \\
\text { di-glucopyranoside }\end{array}$ & Lignans & He et al., 2018 \\
\hline 50 & 13.516 & $\mathrm{C}_{28} \mathrm{H}_{36} \mathrm{O}_{13}$ & 579.2027 & & $\begin{array}{l}151.0031 \\
166.0262, \\
181.0499 \\
402.1316 \\
417.1558\end{array}$ & 1.92 & Syringaresinol 4'-o-glucopyranoside & Lignans & $\begin{array}{l}\text { He et al., 2018; Qi } \\
\text { et al., } 2019\end{array}$ \\
\hline
\end{tabular}

(Continued) 
TABLE 1 | Continued

\begin{tabular}{|c|c|c|c|c|c|c|c|c|c|}
\hline $\begin{array}{l}\text { Cpd } \\
\text { no. }\end{array}$ & $\begin{array}{c}\mathbf{R t} \\
(\mathrm{min})\end{array}$ & Formula & [M-H]- & $\begin{array}{c}{[\mathrm{M}} \\
+\mathrm{COOH}]-\end{array}$ & MS/MS(-) & $\Delta$ ppm & Identification & & References \\
\hline 51 & 13.604 & $\mathrm{C}_{27} \mathrm{H}_{34} \mathrm{O}_{12}$ & 549.1978 & & $\begin{array}{l}151.0398 \\
181.0498 \\
372.1203 \\
387.1439 \\
150.0335\end{array}$ & -4.09 & Eucommin A & Lignans & $\begin{array}{l}\text { He et al., 2018; Qi } \\
\text { et al., } 2019\end{array}$ \\
\hline 52 & 13.859 & $\mathrm{C}_{20} \mathrm{H}_{22} \mathrm{O}_{7}$ & 373.1278 & & $\begin{array}{l}162.0344 \\
165.0574\end{array}$ & 3.95 & Threo-guaiacylglyc erol- $\beta$ conifery aldehyde ether & Lignans & He et al., 2018 \\
\hline 53 & 13.875 & $\mathrm{C}_{25} \mathrm{H}_{24} \mathrm{O}_{12}$ & 515.1189 & & $\begin{array}{l}135.0442, \\
155.0344 \\
161.0224 \\
173.0448 \\
179.0342 \\
191.0557 \\
335.0774 \\
353.0874\end{array}$ & 1.16 & Isochlorogenic acid C & Phenylpropanoids & $\begin{array}{l}\text { He et al., } \\
\text { 2018; Jia et al., } \\
2019\end{array}$ \\
\hline 54 & 14.063 & $\mathrm{C}_{42} \mathrm{H}_{52} \mathrm{O}_{21}$ & 891.2918 & & $\begin{array}{l}167.0396, \\
311.0770 \\
387.1431 \\
417.1552\end{array}$ & 1.16 & Syringaresinol vanillic acid ether diglucopyranoside & Lignans & $\begin{array}{l}\text { He et al., } \\
2018 \text {; Jia et al., } \\
2019\end{array}$ \\
\hline 55 & 14.195 & $\mathrm{C}_{40} \mathrm{H}_{48} \mathrm{O}_{19}$ & 831.2689 & & $\begin{array}{l}167.0379 \\
311.0753 \\
343.1194 \\
519.1846 \\
669.2187\end{array}$ & 3.37 & Pinoresinol vanillic acid ether & Lignans & $\begin{array}{l}\text { He et al., } \\
\text { 2018; Jia et al., } \\
2019\end{array}$ \\
\hline 56 & 14.264 & $\mathrm{C}_{15} \mathrm{H}_{26} \mathrm{O}_{7}$ & 317.1601 & & $\begin{array}{l}163.1155 \\
181.1225 \\
199.1346 \\
207.1019 \\
225.1139 \\
243.1235\end{array}$ & 1.50 & $\begin{array}{l}\text { diglucopyranoside } \\
\text { 2-(5-Hydroxy-2,3-dimethyl-2- cyclopenten-1-yl) } \\
\text { ethylglucopyranoside }\end{array}$ & Others & He et al., 2018 \\
\hline 57 & 14.265 & $\mathrm{C}_{41} \mathrm{H}_{50} \mathrm{O}_{20}$ & 861.2794 & & $\begin{array}{l}151.0400 \\
311.0760 \\
357.1375 \\
387.1440 \\
699.2325\end{array}$ & 3.33 & Medioresinol vanillic acid ether diglucopyranoside & Lignans & $\begin{array}{l}\text { He et al., } \\
\text { 2018; Jia et al., } \\
2019\end{array}$ \\
\hline 58 & 14.334 & $\mathrm{C}_{20} \mathrm{H}_{22} \mathrm{O}_{7}$ & 373.1295 & & $\begin{array}{l}136.0157 \\
181.0514 \\
188.0471 \\
269.0825 \\
285.1125 \\
298.0855 \\
313.1083 \\
358.1049\end{array}$ & -0.60 & 1-Hydroxypinoresinol & Lignans & $\begin{array}{l}\text { Pi et al., } \\
\text { 2016; He et al., } \\
\text { 2018; Jiang et al., } \\
2019\end{array}$ \\
\hline 59 & 14.398 & $\mathrm{C}_{21} \mathrm{H}_{24} \mathrm{O}_{7}$ & & 433.1499 & $\begin{array}{l}166.0253 \\
181.0472\end{array}$ & 1.30 & Medioresinol & Lignans & $\begin{array}{l}\text { He et al.,2018; } \\
\text { Jia et al.,2019 }\end{array}$ \\
\hline 60 & 14.401 & $\mathrm{C}_{40} \mathrm{H}_{48} \mathrm{O}_{19}$ & 831.2686 & & $\begin{array}{l}167.0336 \\
311.0764 \\
343.1177\end{array}$ & 3.75 & Pinoresinol vanillic acid ether di-o-glucoside isomer & Lignans & $\begin{array}{l}\text { He et al., 2018; } \\
\text { Jiang et al., } 2019\end{array}$ \\
\hline 61 & 14.604 & $\mathrm{C}_{37} \mathrm{H}_{46} \mathrm{O}_{16}$ & 745.2690 & & $\begin{array}{l}151.0388 \\
165.0550 \\
181.0477 \\
195.0643 \\
341.0859 \\
357.1361 \\
387.1446 \\
535.1957 \\
583.2191\end{array}$ & 3.09 & Glycerol-medioresinol ether 4"- glucopyranoside & Lignans & $\begin{array}{l}\text { He et al., } \\
\text { 2018; Jiang et al., } \\
\text { 2019; Jia et al., } \\
2019\end{array}$ \\
\hline 62 & 15.094 & $\mathrm{C}_{37} \mathrm{H}_{46} \mathrm{O}_{16}$ & 745.2688 & & $\begin{array}{l}165.0549 \\
181.0494 \\
195.0654 \\
357.1359 \\
387.1438\end{array}$ & -4.51 & Glycerol-medioresinol ether 4"'-glucopyranoside & Lignans & $\begin{array}{l}\text { He et al.,2018; } \\
\text { Jiang et al., } \\
\text { 2019; Jia et al., } \\
2019\end{array}$ \\
\hline
\end{tabular}

(Continued) 
TABLE 1 | Continued

\begin{tabular}{|c|c|c|c|c|c|c|c|c|c|}
\hline $\begin{array}{l}\text { Cpd } \\
\text { no. }\end{array}$ & $\underset{(\mathrm{min})}{\mathrm{Rt}}$ & Formula & {$[\mathrm{M}-\mathrm{H}]-$} & $\begin{array}{l}{[\mathrm{M}} \\
+\mathrm{COOH}]-\end{array}$ & MS/MS(-) & $\Delta \mathrm{ppm}$ & Identification & & References \\
\hline & & & & & $\begin{array}{l}505.1856 \\
535.1964 \\
583.2174\end{array}$ & & & & \\
\hline 63 & 16.3 & $\mathrm{C}_{36} \mathrm{H}_{42} \mathrm{O}_{16}$ & 729.2393 & & $\begin{array}{l}167.0367 \\
181.0493 \\
311.0771 \\
341.0870 \\
387.1440 \\
403.1367 \\
417.1560\end{array}$ & 0.38 & Syringaresinol vanillic acid ether glucopyranoside & Lignans & $\begin{array}{l}\text { He et al., } \\
2018\end{array}$ \\
\hline 64 & 16.383 & $\mathrm{C}_{35} \mathrm{H}_{40} \mathrm{O}_{15}$ & 699.2292 & & $\begin{array}{l}151.0388, \\
167.0342, \\
197.0446, \\
311.0778, \\
341.0881 \\
357.1340 \\
387.1440\end{array}$ & 0.35 & Medioresinol vanillic acid ether glucopyranoside & Lignans & $\begin{array}{l}\text { He et al., } \\
\text { 2018; Jiang et al., } \\
\text { 2019; Jia et al., } \\
2019\end{array}$ \\
\hline 65 & 16.586 & $\mathrm{C}_{34} \mathrm{H}_{38} \mathrm{O}_{14}$ & 669.2182 & & $\begin{array}{l}167.0352, \\
311.0772, \\
327.1228, \\
343.1191 \\
357.1316\end{array}$ & 1.01 & Pinoresinol vanillic acid ether glucopyranoside & Lignans & $\begin{array}{l}\text { He et al., } \\
\text { 2018; Jia et al., } \\
2019\end{array}$ \\
\hline 66 & 16.638 & $\mathrm{C}_{9} \mathrm{H}_{16} \mathrm{O}_{3}$ & 171.1023 & & 153.0902 & 0.35 & 1-Deoxyeucommiol & Iridoids & $\begin{array}{l}\text { He et al., } \\
2018\end{array}$ \\
\hline 67 & 17.873 & $\mathrm{C}_{12} \mathrm{H}_{20} \mathrm{O}_{4}$ & 227.1281 & & $\begin{array}{l}143.8607 \\
165.1225\end{array}$ & 3.43 & $\begin{array}{l}\text { 5,6,7,8-tetrahydro-7-hydroxy-3,3- dimethyl-1H- } \\
\text { cyclopenta[1,3]dioxepin-6-ethanol }\end{array}$ & Others & $\begin{array}{l}\text { He et al., } \\
2018\end{array}$ \\
\hline
\end{tabular}

Although several lignans such as compounds 25, 30, 34, 43, 44, and 49 could not be found based on the characteristic ions, they were also tentatively identified by comparing with the precise parent ions (error below $5 \mathrm{ppm}$ ), $\mathrm{MS}^{2}$ fragment ions and the retention behavior with the data obtained in literatures (He et al., 2018; Jiang et al., 2019).

\section{Identification of Phenylpropanoids}

The phenylpropanoids in Eucommiae Cortex were divided into the simple phenylpropanoids and polyol phenylpropanoids, that is, caffeoyl quinic acids. In general, the caffeoyl quinic acids were more prone to produce [caffeoyl] ${ }^{-}$ion peak at m/z 179 or/and [quinine] ${ }^{-}$ion peak at $\mathrm{m} / \mathrm{z} 191$ (Ouyang et al., 2017; He et al., 2018; Jiang et al., 2019). The $\mathrm{MS}^{2}$ ion peak at $\mathrm{m} / \mathrm{z} 173$ appeared because one molecule $\mathrm{H}_{2} \mathrm{O}$ was separated from the precursor ion at m/z 191 (Özgen et al., 2009; He et al., 2018; Jiang et al., 2019). Thus, the characteristic diagnosis ion at 191, 179, and/or 173 were used for rapid identification of compounds $11,15,17,18$, 22, 31, 41, and 53. Compounds 11, 17, and 18 exhibited the same molecular ion $[\mathrm{M}-\mathrm{H}]^{-}$at $\mathrm{m} / \mathrm{z} 353$ and also shared the product ions at $\mathrm{m} / \mathrm{z} 161$ and 135 attributed to the loss of one molecular $\mathrm{H}_{2} \mathrm{O}(18 \mathrm{Da})$ and $\mathrm{CO}_{2}(44 \mathrm{Da})$ from the [caffeoyl] ${ }^{-}$ion at $\mathrm{m} / \mathrm{z}$ 179. According to the information reported, compounds 11, 17, and $\mathbf{1 8}$ were tentatively speculated as neochlorogenic acid, chlorogenic acid, and cryptochlorogenic acid (Allen et al., 2015; He et al., 2018; Jia et al., 2019), respectively. The cleavage pattern of isomers $\mathbf{4 1}$ and $\mathbf{5 3}$ were basically consistent with chlorogenic acid isomers. Therefore, it was inferred that compounds 41 and 53 were isochlorogenic acid A and $\mathrm{C}(\mathrm{He}$ et al., 2018; Jia et al., 2019), respectively. In addition, the simple phenylpropanoids (compounds 4 and 19) in Eucommiae Cortex were cleaved in the different way. The $[\mathrm{M}-\mathrm{H}]^{-}$ion of compound 19 (caffeic acid) at $\mathrm{m} / \mathrm{z} 179$ produced an $\left[\mathrm{M}-\mathrm{H}-\mathrm{CO}_{2}\right]^{-}$ion at $\mathrm{m} / \mathrm{z}$ 135 and an $\left[\mathrm{M}-\mathrm{H}-\mathrm{CO}_{2}-\mathrm{H}_{2} \mathrm{O}\right]^{-}$ion at $\mathrm{m} / \mathrm{z} 117$. However, the product ion [M-H-Glc] $]^{-}$of compound 4 (protocatechuicacid-4glucoside) at $\mathrm{m} / \mathrm{z} 153$ eliminated the neutral group $\mathrm{CO}_{2}$ to yield the ion at $\mathrm{m} / \mathrm{z} 108$ (Zhang et al., 2016; He et al., 2018).

\section{Identification of Iridoids}

A total of 10 iridoids were identified in this work, including compounds 2, 3, 8, 12, 23, 27, 38, 40, 48, and 66 (Özgen et al., 2009; Allen et al., 2015; Pi et al., 2016; He et al., 2018; Hsueh and Tsai, 2018; Jiang et al., 2019). The most iridoid glycosides were inclining to get aglycon ion due to eliminate glucose neutral group (162 Da). For example, compounds 2, 3, 8, and 23 yielded [M-H-Glc] $]^{-}$ion at m/z 183, 227, 211 and 225 (Allen et al., 2015; He et al., 2018; Jia et al., 2019; Jiang et al., 2019). As reported in the literatures (He et al., 2018; Jiang et al., 2019), the characteristic ions of iridoids were at m/z 101, 119 and/or 147. The identified iridoids except for 38 48, and 66 showed the characteristic diagnosis ions at m/z 101 and m/z 147 (He et al., 2018). The characteristic ion at $\mathrm{m} / \mathrm{z} 101$ was indicative of a $\mathrm{CH}_{2} \mathrm{OH}$ group or $\mathrm{CH}_{3}$ and $\mathrm{OH}$ groups linked to the $\mathrm{C}-8$ position. Another characteristic ion at $\mathrm{m} / \mathrm{z} \mathrm{147}$, was the consequence of successive elimination of glycosidic moiety or loss of $\mathrm{H}_{2} \mathrm{O}, \mathrm{CO}_{2}, \mathrm{HCOOH}$, and $\mathrm{HCOOCH}_{3}$ moiety. The 


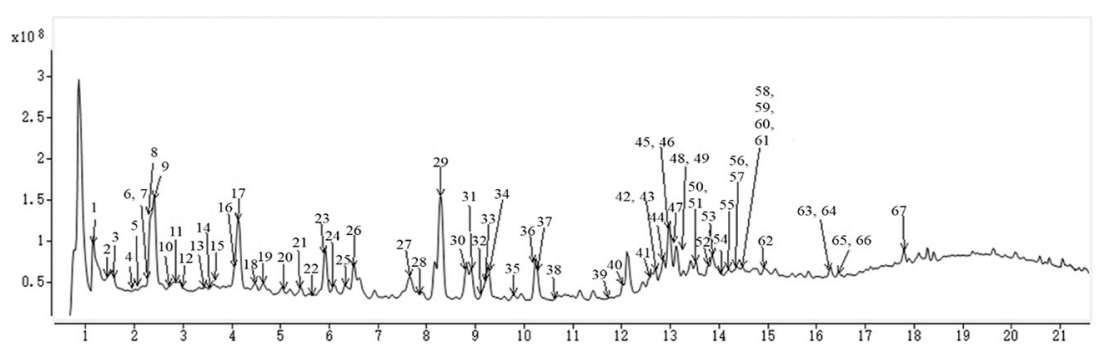

FIGURE 1 | The total ion chromatograms of Eucommiae Cortex by ultra-high performance liquid chromatography coupled with mass spectrometry (UHPLC-Q-TOF/ MS) in negative ion mode.

compounds 38, 48, and 66 displayed $(\mathrm{M}-\mathrm{H})^{-}$ions at $\mathrm{m} / \mathrm{z} 217,187$ and 171 corresponding to chemical formula $\mathrm{C}_{10} \mathrm{H}_{18} \mathrm{O}_{5}, \mathrm{C}_{9} \mathrm{H}_{16} \mathrm{O}_{4}$, and $\mathrm{C}_{9} \mathrm{H}_{16} \mathrm{O}_{3}$. Their $\mathrm{MS}^{2}$ ions peak at $\mathrm{m} / \mathrm{z} 199,169$, and 153 were yielded by loss of $\mathrm{H}_{2} \mathrm{O}$ from the parent ion. Moreover, the other $\mathrm{MS}^{2}$ ions and retention behavior were consistent with that in the reported literatures (He et al., 2018; Jia et al., 2019). Thus, compounds 38, 48, and 66 were probably epieucommiol, eucommiol and 1-deoxyeucommiol.

\section{Identification of Phenolic Acids}

Compounds 6, 7, 9, 10, 20, and 32 (Table 1) were tentatively identified on the basis of the key ions at $\mathrm{m} / \mathrm{z} 123$ and 153 indicative of the core skeleton similar to derivatives of catechol and 3,4-dihydroxy benzoic acid (He et al., 2018; Lei et al., 2018; Jiang et al., 2019; Jia et al., 2019). Moreover, a few of common neutral fragments such as $\mathrm{CH}_{3}, \mathrm{CO}_{2}$, and glucosyl unit were also recognized as important identification features of phenolic acids. For example, the vanillic acid (compounds 7) lose the methyl radical $\left(\mathrm{CH}_{3}\right)$ and one molecule $\mathrm{CO}_{2}$ to get fragment ions at $\mathrm{m} / \mathrm{z}$ 152 and $\mathrm{m} / \mathrm{z}$ 108, respectively (Lei et al., 2018).

\section{Identification of Other Compounds}

As to other compounds $(1,5,13,14,21,26,33,39,56$, and 67$)$, it was impossible to identify compounds base on the key characteristic ions due to the lack of detail information about shared structure. However, the compounds could be tentatively identified by comparing the experimental data with information of literatures, such as precise MS data and fragment ions ( $\mathrm{He}$ et al., 2018; Jia et al., 2019; Jiang et al., 2019).

\section{Metabolomics Data Analysis}

Metabolomics analysis has good performance on screening the difference compounds in natural plant samples. Using the $\mathrm{R}$ package XCMS, all the raw mass spectra data of C1-C19 and S1S19 samples, which were acquired from UHPLC-Q-TOF/MSESI$^{-}$, was converted into a three-dimensional matrix including information of a mass of variables, such as retention times, $\mathrm{m} / \mathrm{z}$ values, peak intensities. Then 2,843 variables were generated and were subjected to OPLS-DA analysis on the SIMIC software. OPLS-DA, a supervised multivariate data analysis method, was characterized by difference analysis of inter-groups. The OPLSDA plot (Figure 2A) displayed the obvious separation between crude and salt-fired samples in the presence of 2,843 variables.
However, 2,843 variables were not practical for distinction of two types of Eucommiae Cortex and even QC assessment. Thus OPLS-DA was further utilized to mine potential and obvious difference compounds based on the value of variable importance parameter (VIP) higher than 1, which was considered to greatly contribute to the separation of clustering. Then a total of 505 candidate compounds were rapidly filtered from 2,843 variables. It was shown (Figure 2B) that the crude and salt-fired Eucommiae Cortex was well distinguished by the 505 compounds as candidate markers.

\section{Identification of the Candidate Markers}

The 505 candidate markers with the VIP $>1$ would be explicitly identified on the basis of qualitative study of compounds in Eucommiae Cortex. Thirty-seven compounds were rapidly identified from 505 candidate markers according to $\mathrm{m} / \mathrm{z}$ values and retention time of the significant difference markers. They were respectively compounds $1,2,3,5,6,8,9,10,11,14,16,17$, $19,21,23,25,26,27,29,33,34,37,39,40,41,43,44,46,48,49$, $50,53,60,63,64,65$, and 66 (Table 1). The other unknown markers would continue to be identified. Moreover, OPLS-DA analysis results (Figure 2C) showed that two groups of Eucommiae Cortex samples were basically differentiated by the 37 candidate markers. It suggested that the filtered 37 compounds might be potential CdQMs as an alternative to the 505 candidate markers.

\section{Selection and Verification of the Final CdQMs}

Although the range of difference markers was limited to 37 CdQMs in Eucommiae Cortex by the analysis of OPLS-DA, it was still considerably difficult to simultaneously achieve the QC and effective distinction of the crude Eucommiae Cortex and its salt-fired product. Therefore, it was indeed necessary to further filter the practical CdQMs from the above 37 identified CdQMs. Then the CdQMs would be unambiguously defined according to the following characteristics: easy quantitation, commercial access, and the most importantly, good distinction ability to two types of Eucommiae Cortex products. Consequently, eleven compounds (geniposidic acid, neochlorogenic acid, chlorogenic acid, caffeic acid, geniposide, genipin, pinoresinol di-oglucopyranoside, syringaresinol di-o-glucopyranoside, isochlorogenic acid $\mathrm{A}$, and isochlorogenic acid $\mathrm{C}$ ) were roughly 

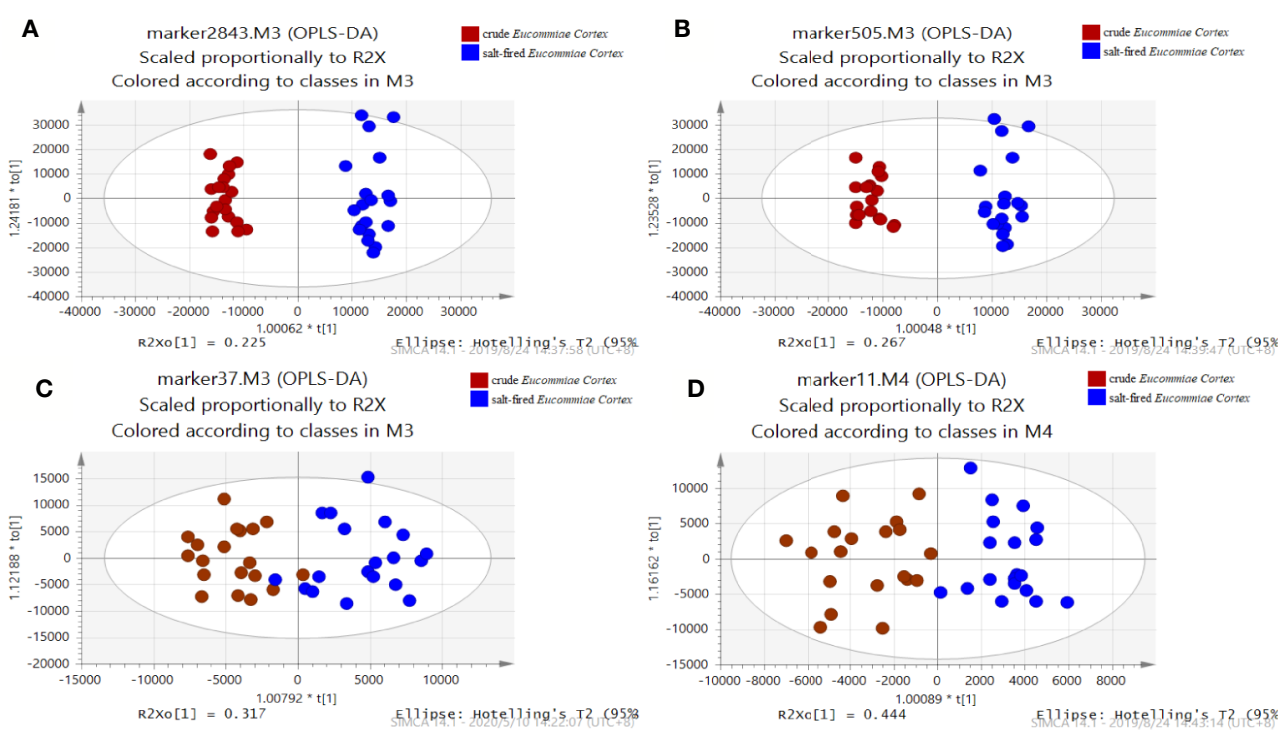

FIGURE 2 | The orthogonal partial least-squares discriminant analysis (OPLS-DA) model for 38 samples of the crude and salt-fired Eucommiae Cortex by 2,843 variables (A), 505 variables (B), 37 variables (C), and 11 variables (D), respectively.

selected as potential CdQMs based on the first two characteristics. The OPLS-DA analysis (Figure 2D) showed that 11 potential CdQMs could well separate the crude samples and salt-fired samples. However, the accuracy of the selected CdQMs needs to be further validated. Herein, two supervised learning model, the PLSR and RF, were implemented to determine the accuracy of the markers generated via each filtering steps. The batches of C1-10 and S1-10 were respectively set as training set of the crude group and the saltfired group. The remaining batches (C11-19 of crude Eucommiae Cortex and S11-19 of salt-fired Eucommiae Cortex) were analyzed as testing set. In general, the training set was used to build a model, whereas the testing set was used to verify the established model and provide the accuracies of related variables. Finally, the PLSR and RF algorithms were employed to predict and classify the 38 batches of samples with the $2843,505,37$, and 11 compounds as variables, respectively. The analysis results of algorithms (Table 2) showed the accuracies of 2,843, 505, and 11 variables were all more than $90 \%$, whereas the accuracy of 37 variables were obviously lower than those of others. It demonstrated that the 37 compounds were not optimal candidate markers. Interestingly, the accuracy of 11 variables was equivalent to that of 505 variables, and even close to the accuracy of 2,843 variables. Therefore, the 11 compounds as CdQMs could be fully behalf of the whole compounds in Eucommiae Cortex for distinguishing the crude and salt-fired Eucommiae Cortex and were used for quality evaluation of twotypes of Eucommiae Cortex products.

\section{UHPLC-PDA Quantitative Method Validation}

To validate the UHPLC-PDA method, the selectivity, linearity, LOD and LOQ, repeatability, accuracies and precisions, stability, and recoveries should be investigated and the related data was well displayed (Tables S3 and S4). In contrast to the chromatogram of the 11 standard substances and blank solution, obvious interference was not observed in the chromatogram of extract solution (Figure 3), indicating that the analytical method had good selectivity for detection of 11 analytes. A total of 11 standard curve lines enabled to accurately determine the concentrations of target components within the analysis range due to the $\mathrm{r}^{2}$ values more than 0.9991 . The range of LOQs and LODs for 11 CdQMs were from 0.03 to $1.00 \mu \mathrm{g} / \mathrm{ml}$ and 0.01 to $0.3 \mu \mathrm{g} / \mathrm{ml}$, respectively. The detection method was much stable to determine multisamples due to the RSDs of repeatability below $5 \%$. The intra-day and inter-day accuracies were the range of $88.2 \%-105 \%$ and the RSDs of the corresponding precisions were within $0.10 \%-4.69 \%$. The results obviously validated the fact that this quantitative method could analyze accurately the samples in several days. The recoveries of this method for the 11 components ranged from $95.0 \%$ to $104 \%$ (Table S3), fully demonstrating the extremely little loss of target compounds in the extraction and sampling process. Overall, this developed UHPLC-PDA method was well fitting for the analysis of the 11 CdQMs in Eucommiae Cortex samples.

TABLE 2 | The accuracies of different variables by PLSR and RF Algorithms.

\begin{tabular}{lcccc}
\hline Algorithms & \multicolumn{4}{c}{ Different amounts of variables } \\
\cline { 2 - 5 } & $\mathbf{2 8 4 3}$ & $\mathbf{5 0 5}$ & $\mathbf{3 8}$ & $\mathbf{1 1}$ \\
\hline PLSR & $100 \%$ & $100 \%$ & $66.7 \%$ & $94.1 \%$ \\
RF & $100 \%$ & $100 \%$ & $83.3 \%$ & $94.1 \%$
\end{tabular}




\section{Analysis of Different Batches of Eucommiae Cortex samples}

In order to exclude the influence of origin places on the selection of quality markers, 8 batches of the crude Eucommiae Cortex and their salt-fired products from Sichuan Province in China were analyzed using the same OPLS-DA strategy according to the same rules. The same eleven quality markers were also found and filtered. Although VIP values of eleven quality markers (Table S5) from the same origin place were different with those of samples from the different origin places, these eleven quality markers could divide these samples into two groups. One is the crude and the other is salt-fired group. This result was basically consistent with the real situation. Thus, the processing could change the chemical contents of 11 CdQMs in crude samples leading to the difference from the salt-fired samples. Based on the above analysis, eleven CdQMs were identified and regarded as the featured markers that could be alternative to the whole chemical compounds profile for differentiation of the crude and the salt-fired Eucommiae Cortex.

The validated UHPLC-PDA method was employed to simultaneously determine the content of the $11 \mathrm{CdQMs}$ (geniposidic acid, neochlorogenic acid, chlorogenic acid, caffeic acid, geniposide, genipin, pinoresinol di-o-glucopyranoside, syringaresinol di-o-glucopyranoside, isochlorogenic acid A, pinoresinol o-glucopyranoside, and isochlorogenic acid C) in 54 batches of Eucommiae Cortex samples. Among them, the C1C19 and VC1-VC8 batches were crude Eucommiae Cortex and remaining batches (S1-S19 and VS1-VS8) were salt-fired Eucommiae Cortex. Base on the average content of each marker (Table 3), the contents of nine markers (geniposidic acid, neochlorogenic acid, caffeic acid, geniposide, genipin, pinoresinol di-o-glucopyranoside, syringaresinol di-oglucopyranoside, isochlorogenic acid $\mathrm{A}$, and isochlorogenic acid C) were reduced while two markers (chlorogenic acid and
TABLE 3 | The average content of 11 CdQMs in different bathes of Eucommiae Cortex samples $(\mu \mathrm{g} / \mathrm{mg})$.

\begin{tabular}{llllllllllll}
\hline \multirow{2}{*}{ Batches } & \multicolumn{10}{c}{ Average content $(\mu \mathrm{g} / \mathbf{m g})$} \\
\cline { 2 - 11 } & M1 & M2 & M3 & M4 & M5 & M6 & M7 & M8 & M9 & M10 & M11 \\
\hline C & 1.65 & 0.06 & 0.51 & 0.05 & 0.31 & 0.40 & 1.81 & 0.52 & 0.16 & 0.32 & 0.04 \\
S & 1.45 & 0.05 & 0.54 & 0.02 & 0.28 & 0.08 & 1.22 & 0.30 & 0.13 & 0.36 & 0.03
\end{tabular}

M1-11 represented geniposidic acid, neochlorogenic acid, chlorogenic acid, caffeic acid, geniposide, genipin, pinoresinol di-o-glucopyranoside, syringaresinol di-oglucopyranoside, isochlorogenic acid A, pinoresinol o-glucopyranoside, and isochlorogenic acid C, respectively; C represented the crude Eucommiae Cortex group (C1-C19 and VC1-VC8); S represented the salt-fired Eucommiae Cortex group (S1-S19 and VS1-VS8).

pinoresinol $o$-glucopyranoside) were increased after crude Eucommiae Cortex samples were salt-fired. The possible reason was relation to the structure transformation of compounds such as oxidation, decomposition, isomerization in the salt-fired process ( Wu et al., 2018). Moreover, these CdQMs had a variety of pharmacological activities such as antioxidant, antiinflammatory, anti-cancer, anti-atherosclerosis, and antihypertension (Gao et al., 2015; Li et al., 2015; Liu et al., 2016; Wang J. et al., 2017; Ma et al., 2019; Xia et al., 2019). Thus, content fluctuation of these markers between the crude Eucommiae Cortex and its salt-fired product probably lead to change in bioactive effects. Because many factors could affect the content of chemical ingredients in Eucommiae Cortex, more indepth research need to be carried out for clarifying the influence of processing on the multiple chemical ingredients of Eucommiae Cortex in the future.

\section{Discriminant Analysis}

Discriminant analysis was characterized by predicting classification of the unknown sample. Discriminant analysis was used to determine whether the unknown samples are

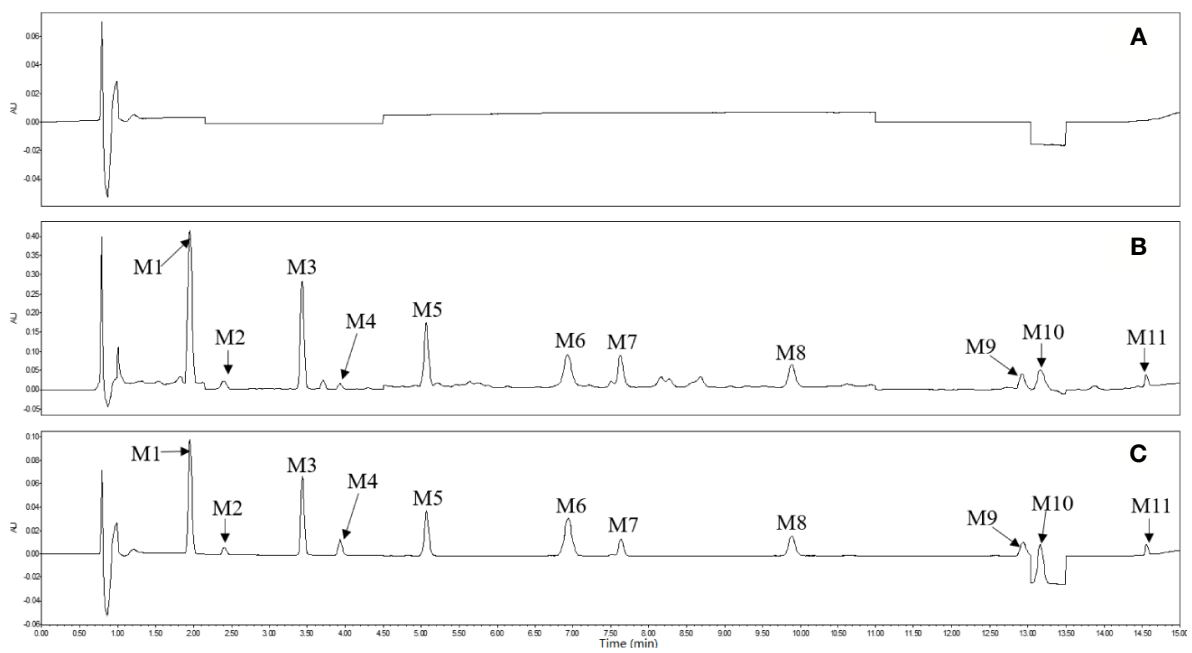

FIGURE 3 | Ultra-high performance liquid chromatography (UHPLC) chromatograms of blank solvent solution (A), sample solution (B), and mixed standard solution (C). M1-11 represented geniposidic acid, neochlorogenic acid, chlorogenic acid, caffeic acid, geniposide, genipin, pinoresinol di-o-glucopyranoside, syringaresinol dio-glucopyranoside, isochlorogenic acid A, pinoresinol o-glucopyranoside, and isochlorogenic acid C, respectively. 
TABLE 4 | The classification results by discriminant analysis.

\begin{tabular}{|c|c|c|c|}
\hline Batches & Actual groups & Predictive groups & Discriminant scores \\
\hline C1 & 1 & 1 & 1.55389 \\
\hline $\mathrm{C} 2$ & 1 & 1 & 0.34026 \\
\hline C3 & 1 & 1 & 2.53401 \\
\hline C4 & 1 & $2^{\#}$ & -0.56361 \\
\hline C5 & 1 & 1 & 1.42251 \\
\hline C6 & 1 & 1 & 1.35273 \\
\hline $\mathrm{C} 7$ & 1 & 1 & 2.13122 \\
\hline $\mathrm{C} 8$ & 1 & 1 & 1.04150 \\
\hline $\mathrm{C9}$ & 1 & 1 & 0.22947 \\
\hline C10 & 1 & 1 & 0.02528 \\
\hline C11 & 1 & 1 & 2.49143 \\
\hline C12 & 1 & 1 & 1.80828 \\
\hline $\mathrm{C} 13$ & 1 & 1 & 3.15104 \\
\hline C14 & 1 & 1 & 0.44475 \\
\hline C15 & 1 & 1 & 0.72497 \\
\hline C16 & 1 & 1 & 2.80181 \\
\hline C17 & 1 & 1 & 0.71904 \\
\hline C18 & 1 & 1 & 1.33373 \\
\hline C19 & 1 & 1 & 2.97135 \\
\hline S1 & 2 & 2 & -1.73808 \\
\hline S2 & 2 & 2 & -0.53112 \\
\hline S3 & 2 & 2 & -1.16221 \\
\hline S4 & 2 & 2 & -0.71001 \\
\hline S5 & 2 & 2 & -0.45801 \\
\hline S6 & 2 & 2 & -1.39650 \\
\hline S7 & 2 & 2 & -1.94982 \\
\hline S8 & 2 & 2 & -2.20743 \\
\hline s9 & 2 & 2 & -1.21746 \\
\hline S10 & 2 & 2 & -1.99632 \\
\hline S11 & 2 & 2 & -1.10804 \\
\hline S12 & 2 & 2 & -2.16652 \\
\hline S13 & 2 & 2 & -0.07373 \\
\hline S14 & 2 & 2 & -3.69995 \\
\hline S15 & 2 & 2 & -0.25776 \\
\hline S16 & 2 & 2 & -1.15230 \\
\hline S17 & 2 & 2 & -0.71390 \\
\hline S18 & 2 & 2 & -1.20901 \\
\hline S19 & 2 & 2 & -2.76548 \\
\hline VC1 & - & 1 & 0.54668 \\
\hline VC2 & - & 1 & 1.73195 \\
\hline VC3 & - & 1 & 1.58948 \\
\hline VC4 & - & 1 & 1.96910 \\
\hline VC5 & - & 1 & 1.16438 \\
\hline VC6 & - & 1 & 1.64134 \\
\hline VC7 & - & 1 & 3.28505 \\
\hline VC8 & - & 1 & 0.39950 \\
\hline VS1 & - & 2 & -0.08497 \\
\hline VS2 & - & 2 & -0.46013 \\
\hline VS3 & - & 2 & -0.68333 \\
\hline VS4 & - & 2 & -0.07023 \\
\hline VS5 & - & 2 & -1.09459 \\
\hline VS6 & - & 2 & -3.20495 \\
\hline VS7 & - & $1^{\#}$ & 1.26397 \\
\hline VS8 & - & 2 & -0.07440 \\
\hline
\end{tabular}

1, represented the crude Eucommiae Cortex group; 2, represented the salt-fired Eucommiae Cortex group; -, represented the unknown groups; "represented the incorrect classification.

crude or salt-fired Eucommiae Cortex. The crude samples (C1C19) and salt-fired samples (S1-S19) were labeled as group 1 and group 2 (Table 4), respectively. The contents of 11 CdQMs in these samples were used as modeling data to build the unstandardized canonical discriminant model by SPSS software. The samples (VC1-VC8 and VS1-VS8) were selected as testing sample. The discriminant function generated was showed as follows:

$$
\begin{aligned}
\gamma= & 0.06 \mathrm{X}_{1}+14.8 \mathrm{X}_{2}-6.16 \mathrm{X}_{3}+6.18 \mathrm{X}_{4}+3.18 \mathrm{X}_{5}+1.65 \mathrm{X}_{6} \\
& +2.37 \mathrm{X}_{7}-0.45 \mathrm{X}_{8}+0.29 \mathrm{X}_{9}-2.91 \mathrm{X}_{10}+1.49 \mathrm{X}_{11}-1.60
\end{aligned}
$$

where $\mathrm{X}_{1}$ to $\mathrm{X}_{11}$ represented the contents of geniposidic acid, neochlorogenic acid, chlorogenic acid, caffeic acid, geniposide, genipin, pinoresinol di-o-glucopyranoside, syringaresinol di-oglucopyranoside, isochlorogenic acid A, pinoresinol $o$ glucopyranoside, and isochlorogenic acid C, respectively; the $\gamma$ is the discriminant score. The classification accuracies of this model were $97.4 \%$ and $78.9 \%$ corresponding to originally grouped cases and cross-validation grouped cases, respectively. It demonstrated that the reliability of this discriminant model was acceptable. Discriminant score of each sample was calculated through the discriminant function. Two centroid values of crude Eucommiae Cortex and salt-fired Eucommiae Cortex group were respectively 1.395 and -1.395 . Their sum was the discriminant value. If discriminant score of one sample was higher than 0 , it would be classified into the crude Eucommiae Cortex group. Otherwise, they would belong to salt-fired Eucommiae Cortex. The results of predictive groups (Table 4) displayed that most of samples except for C4 in known groups were correctly classified. Only one unclassified sample (VS7) were not correctly predicted. These results demonstrated that simultaneous determination of 11 CdQMs coupled with discriminant analysis could well be used to differentiate the crude and salt-fired Eucommiae Cortex.

\section{CONCLUSION}

The CdQMs were screened for precise quality assessment of crude and salt-fired Eucommiae Cortex by LC-MS metabolomics with chemometrics strategy. An in-house component library of Eucommiae Cortex was built for rapid search of known compounds, which would make the qualitative analysis efficient and time-saving. Eleven CdQMs including geniposidic acid, neochlorogenic acid, chlorogenic acid, caffeic acid, geniposide, genipin, pinoresinol di-o-glucopyranoside, syringaresinol di-o-glucopyranoside, isochlorogenic acid A, pinoresinol $o$-glucopyranoside, and isochlorogenic acid $\mathrm{C}$, were screened step by step and could well differentiate the crude and salt-fired Eucommiae Cortex. It was concluded that LC-MS metabolomics with chemometrics was a powerful strategy to filter CdQMs for distinguishing the crude and salt-fired Eucommiae Cortex. It would provide a reliable reference for the in-depth investigation of difference between the crude and salt-fired Eucommiae Cortex.

\section{DATA AVAILABILITY STATEMENT}

All datasets generated for this study are included in the article/ Supplementary Material. 


\section{AUTHOR CONTRIBUTIONS}

$\mathrm{Y}-\mathrm{XC}$ and $\mathrm{XG}$ designed the experiment. Y-XC and JG analyzed the experimental data. JG, JL, XY, HW, JH, and EL performed the experiment and wrote the manuscript.

\section{ACKNOWLEDGMENTS}

This work was supported by Science and Technology Program of Tianjin (No.19ZYPTJC00060), Tianjin Research Program of

\section{REFERENCES}

Özgen, U., Kazaz, C., Secen, H., Çaliş, İ., Coşkun, M., and Houghton, P. J. (2009). A novel naphthoquinone glycoside from Rubia peregrina L. Turk. J. Chem. 33, 561-568. doi: 10.3906/kim-0806-28

Allen, F., Greiner, R., and Wishart, D. (2015). Competitive fragmentation modeling of ESI-MS/MS spectra for putative metabolite identification. Metabolomics 11, 98-110. doi: 10.1007/s11306-014-0676-4

Aszyk, J., Byliński, H., Namieśnik, J., and Kot-Wasik, A. (2018). Main strategies, analytical trends and challenges in LC-MS and ambient mass spectrometrybased metabolomics. Trends Anal. Chem. 108, 278-295. doi: 10.1016/ j.trac.2018.09.010

Brenes, M., Hidalgo, F. J., García, A., Rios, J. J., García, P., Zamora, R., et al. (2000). Pinoresinol and 1-acetoxypinoresinol, two new phenolic compounds identified in olive oil. J. Am. Oil Chem. Soc 77, 715-720. doi: 10.1007/s11746-000-0115-4

Chai, X., Wang, Y., Su, Y. F., Bah, A. J., Hu, L., Gao, Y., et al. (2012). A rapid ultra performance liquid chromatography-tandem mass spectrometric method for the qualitative and quantitative analysis of ten compounds in Eucommia ulmodies Oliv. J. Pharm. Biomed. Anal. 57, 52-61. doi: 10.1016/ j.jpba.2011.08.023

Cronquist, A., and Takhtadzhian, A. L. (1981). An integrated system of classification of flowering plants (New York: Columbia University Press).

Deyama, T. (1983). The constituents of Eucommia ulmoides OLIV. I. Isolation of (+)-Medioresinol Di-O- $\beta$-D-glucopyranoside. Chem. Pharm. Bull. 31, 29932997. doi: $10.1248 / \mathrm{cpb} .31 .2993$

Feng, S., Ni, S., and Sun, W. (2007). Preparative isolation and purification of the lignan pinoresinol diglucoside and liriodendrin from the bark of Eucommia ulmoides Oliv. by high speed countercurrent chromatography. J. Liq. Chromatogr. Relat. Technol. 30, 135-145. doi: 10.1080/10826070601036324

Gao, Y., Chen, Z. Y., Liang, X., Xie, C., and Chen, Y. F. (2015). Anti-atherosclerotic effect of geniposidic acid in a rabbit model and related cellular mechanisms. Pharm. Biol. 53, 280-285. doi: 10.3109/13880209.2014.916310

Guo, H., Liu, A. H., Ye, M., Yang, M., and Guo, D. A. (2007). Characterization of phenolic compounds in the fruits of Forsythia suspensa by high-performance liquid chromatography coupled with electrospray ionization tandem mass spectrometry. Rapid Commun. Mass Spectrom. 21, 715-729. doi: 10.1002/ $\mathrm{rcm} .2875$

He, X., Wang, J., Li, M., Hao, D., Yang, Y., Zhang, C., et al. (2014). Eucommia ulmoides Oliv.: ethnopharmacology, phytochemistry and pharmacology of an important traditional Chinese medicine. J. Ethnopharmacol. 151, 78-92. doi: 10.1016/j.jep.2013.11.023

He, M., Jia, J., Li, J., Wu, B., Huang, W., Liu, M., et al. (2018). Application of characteristic ion filtering with ultra-high performance liquid chromatography quadrupole time of flight tandem mass spectrometry for rapid detection and identification of chemical profiling in Eucommia ulmoides Oliv. J. Chromatogr. A 155, 481-491. doi: 10.1016/j.chroma.2018.04.036

Hsueh, T. P., and Tsai, T. H. (2018). Preclinical pharmacokinetics of scoparone, geniposide and rhein in an herbal medicine using a validated LC-MS/MS method. Molecules 23, 2716. doi: 10.3390/molecules 23102716

Jia, J., Liu, M., Wen, Q., He, M., Ouyang, H., Chen, L., et al. (2019). Screening of anti-complement active ingredients from Eucommia ulmoides Oliv. branches and their metabolism in vivo based on UHPLC-Q-TOF/MS/MS. J. Chromatogr. B. 1124, 26-36. doi: 10.1016/j.jchromb.2019.05.029
Application Foundation and Advanced Technology (18JCYBJC95000), Special Program of Talents Development for Excellent Youth Scholars in Tianjin.

\section{SUPPLEMENTARY MATERIAL}

The Supplementary Material for this article can be found online at: https://www.frontiersin.org/articles/10.3389/fphar.2020. 00838/full\#supplementary-material

Jiang, P., Ma, Y., Gao, Y., Li, Z., Lian, S., Xu, Z., et al. (2016). Comprehensive Evaluation of the Metabolism of Genipin-1- $\beta$-d-gentiobioside in Vitro and in Vivo by Using HPLC-Q-TOF. J. Agric. Food Chem. 64, 5490-5498. doi: 10.1021/acs.jafc.6b01835

Jiang, Y., Liu, R., Chen, J., Liu, M., Liu, M., Liu, B., et al. (2019). Application of multifold characteristic ion filtering combined with statistical analysis for comprehensive profiling of chemical constituents in anti-renal interstitial fibrosis I decoction by ultra-high performance liquid chromatography coupled with hybrid quadrupole-orbitrap high resolution mass spectrometry. J. Chromatogr. A. 1600, 197-208. doi: 10.1016/j.chroma.2019.04.051

Kumar, N., Bansal, A., Sarma, G. S., and Rawal, R. K. (2014). Chemometrics tools used in analytical chemistry: An overview. Talanta 123, 186-199. doi: 10.1016/ j.talanta.2014.02.003

Lei, X. Q., Li, G., Cheng, L., Wang, X. L., and Meng, F. Y. (2018). Identification of Ligustici Rhizoma et Radix and its adulterants based on their chemical constituents by UHPLC-Q/TOF-MS combined with data mining. J. Pharm. Biomed. Anal. 154, 123-137. doi: 10.1016/j.jpba.2018.02.053

Li, Y., Han, C., Wang, J., Xiao, W., Wang, Z., Zhang, J., et al. (2014). Investigation into the mechanism of Eucommia ulmoides Oliv. based on a systems pharmacology approach. J. Ethnopharmacol. 151, 452-460. doi: 10.1021/ acs.jafc. $8 \mathrm{~b} 01312$

Li, L., Guo, Y., Zhao, L., Zu, Y., Gu, H., and Yang, L. (2015). Enzymatic hydrolysis and simultaneous extraction for preparation of genipin from bark of eucommia ulmoides after ultrasound, microwave pretreatment. Molecules 20, 18717 18731. doi: 10.3390/molecules201018717

Li, Y., Xie, Y., He, Y., Hou, W., Liao, M., and Liu, C. (2019). Quality Markers of Traditional Chinese Medicine: Concept, Progress, and Perspective. Engineering 5, 813-980. doi: 10.1016/j.eng.2019.01.015

Liu, E., Lin, Y., Wang, L., Huo, Y., Zhang, Y., Guo, J., et al. (2016). Simultaneous Determination of Pinoresinol Di-glucopyranoside and Pinoresinol Glucoside in Rat Plasma by HPLC-tandem MS/MS for Pharmacokinetic Study. Chin. Herb. Med. 8, 337-343. doi: 10.1016/S1674-6384(16)60060-6

Lu, J., Liu, L., Zhu, X., Wu, L., Chen, Z., Xu, Z., et al. (2018). Evaluation of the Absorption Behavior of Main Component Compounds of Salt-Fried Herb Ingredients in Qing'e Pills by Using Caco-2 Cell Model. Molecules 23, 3321. doi: 10.3390/molecules23123321

Ma, S., Zhang, C., Zhang, Z., Dai, Y., Gu, R., and Jiang, R. (2019). Geniposide protects PC12 cells from lipopolysaccharide-evoked inflammatory injury via up-regulation of miR-145-5p. Artif. Cells Nanomed. Biotechnol. 47, 2875-2881. doi: $10.1080 / 21691401.2019 .1626406$

Mao, Q., Kong, M., Shen, H., Zhu, H., Zhou, S. S., Li, S. L., et al. (2017). LC-MSbased Metabolomics in Traditional Chinese Medicines Research: Personal Experiences. Chin. Herb. Med. 9, 14-21. doi: 10.1016/S1674-6384(17)60071-6

Ouyang, H., Li, J., Wu, B., Zhang, X., Li, Y., Yang, S., et al. (2017). A robust platform based on ultra-high performance liquid chromatography Quadrupole time of flight tandem mass spectrometry with a two-step data mining strategy in the investigation, classification, and identification of chlorogenic acids in Ainsliaea fragrans Champ. J. Chromatogr. A. 1502, 38-50. doi: 10.1016/ j.chroma.2017.04.051

Pi, J. J., Wu, X., Rui, W., Feng, Y. F., and Guo, J. (2016). Identification and fragmentation mechanisms of two kinds of chemical compositions in eucommia ulmoides By UPLC-ESI-Q-TOF-MS/MS. Chem. Nat. Compd. 52, 144-148. doi: 10.1007/s10600-016-1574-y 
Qi, L. W., Chen, C. Y., and Li, P. (2019). Structural characterization and identification of iridoid glycosides, saponins, phenolic acids and flavonoids in Flos Lonicerae Japonicae by a fast liquid chromatography method with diode-array detection and time-of-flight mass spectrometry. Rapid Commun. Mass Spectrom. 23, 3227-3242. doi: 10.1002/rcm.4245

Shi, S. Y., Peng, M. J., Zhang, Y. P., and Peng, S. (2013). Combination of preparative HPLC and HSCCC methods to separate phosphodiesterase inhibitors from Eucommia ulmoides bark guided by ultrafiltration-based ligand screening. Anal. Bioanal. Chem. 405, 4213-4223. doi: 10.1007/s00216-013-6806-4

Wang, F., Wang, B., Wang, L., Xiong, Z. Y., Gao, W., Li, P., et al. (2017). Discovery of discriminatory quality control markers for Chinese herbal medicines and related processed products by combination of chromatographic analysis and chemometrics methods: Radix Scutellariae as a case study. J. Pharm. Biomed. Anal. 138, 70-79. doi: 10.1016/j.jpba.2017.02.004

Wang, J., Cao, G., Wang, H., Ye, H., Zhong, Y., Wang, G., et al. (2017). Characterization of isochlorogenic acid A metabolites in rats using highperformance liquid chromatography/quadrupole time-of-flight mass spectrometry. Biomed. Chromatogr. 31, e3927. doi: 10.1002/bmc.3927

Wang, Y. J., Li, T. H., Jin, G., Wei, Y. M., Li, L. Q., Kalkhajeh, Y. K., et al. (2019). Qualitative and quantitative diagnosis of nitrogen nutrition of tea plants under field condition using hyperspectral imaging coupled with chemometrics. J. Sci. Food. Agric. 100, 161-167. doi: 10.1002/jsfa.10009

Wu, X., Wang, S., Lu, J., Jing, Y., Li, M., Cao, J., et al. (2018). Seeing the unseen of chinese herbal medicine processing (paozhi): advances in new perspectives. Chin. Med. 13, 4. doi: 10.1186/s13020-018-0163-3

Xia, B. H., Hu, Y. Z., Xiong, S. H., Tang, J., Yan, Q. Z., and Lin, L. M. (2017). Application of random forest algorithm in fingerprint of Chinese medicine: different brands of Xiasangju granules as example. China J. Chin. Mater. Med. 42, 1324-1330. doi: 10.19540/j.cnki.cjcmm.20170121.020

Xia, J. X., Zhao, B. B., Zan, J. F., Wang, P., and Chen, L. L. (2019). Simultaneous determination of phenolic acids and flavonoids in Artemisiae Argyi Folium by
HPLC-MS/MS and discovery of antioxidant ingredients based on relevance analysis. J. Pharm. Biomed. Anal. 175, 112734. doi: 10.1016/j.jpba.2019.06.031

Zhang, Q. Q., Dong, X., Liu, X. G., Gao, W., Li, P., and Yan, H. (2016). Rapid separation and identification of multiple constituents in Danhong Injection by ultra-high performance liquid chromatography coupled to electrospray ionization quadrupole time-of-flight tandem mass spectrometry. Chin. J. Nat. Med. 14., 147-160. doi: 10.1016/S1875-5364(16)60008-0

Zhao, B. T., Jeong, S. Y., Kim, T. I., Seo, E. K., Min, B. S., Son, J. K., et al. (2015) Simultaneous quantitation and validation of method for the quality evaluation of Eucommiae cortex by HPLC/UV. Arch. Pharm. Res. 38, 2183-2192. doi: 10.1007/s12272-015-0642-3

Zhou, B., Xiao, J. F., Tuli, L., and Ressom, H. W. (2012). LC-MS-based metabolomics. Mol. Biosyst. 8, 470-481. doi: 10.1039/c1mb05350g

Zhu, M. Q., and Sun, R. C. (2018). Eucommia ulmoides Oliver: a potential feedstock for bioactive products. J. Agric. Food Chem. 66, 5433-5438. doi: 10.1021/acs.jafc.8b01312

Ziegel, E. R. (2004). Statistics and chemometrics for analytical chemistry: statistics and chemometrics for analytical chemistry. Technometrics 46, 498-499. doi: 10.1198/tech.2004.s248

Conflict of Interest: The authors declare that the research was conducted in the absence of any commercial or financial relationships that could be construed as a potential conflict of interest.

Copyright (c) 2020 Guo, Li, Yang, Wang, He, Liu, Gao and Chang. This is an openaccess article distributed under the terms of the Creative Commons Attribution License (CC BY). The use, distribution or reproduction in other forums is permitted, provided the original author(s) and the copyright owner(s) are credited and that the original publication in this journal is cited, in accordance with accepted academic practice. No use, distribution or reproduction is permitted which does not comply with these terms. 